BONPLANDIA 20(2). 2011

ISSN: 0524-0476

\title{
HUERTOS FAMILIARES EN LA BAHÍA DE CISPATÁ, CÓRDOBA, COLOMBIA
}

\author{
NÉSTOR DAVID JIMÉNEZ-ESCOBAR ${ }^{1}$, ULYSSES P. ALBUQUERQUE ${ }^{2} \&$ J. ORLANDO RANGEL-CH. ${ }^{3}$
}

Summary: Jiménez-Escobar, N. D., U. P. Albuquerque \& J. O. Rangel-Ch. 2011. Homegardens in the bay of Cispatá, Córdoba, Colombia. Bonplandia 20(2): 309-328.

\begin{abstract}
Ethnobotanical studies of communities that inhabit estuarine areas and use the mangrove forest are limited in Colombia. In order to support management and conservation projects in the future, we studied family homegardens in rural communities in the bay of Cispatá, Colombian Caribbean. We analyzed structure, floristic composition and uses of tree species in homegardens. In a total area of $29950 \mathrm{~m}^{2}$, where twelve homegardens are located, we recorded 1798 individuals (DAP $\geq 2.5 \mathrm{~cm}$ ) belonging to 83 tree and palm species, corresponding to 32 plant families. The family Leguminosae s.l. was represented by the greatest number of species (12), followed by Annonaceae, Arecaceae and Moraceae (6). Food was the use-category with the highest number of species (46), followed by Medicine (34) and Construction (33). According to the Salience Index in the homegardens Cocos nucifera is the most important species. We found a positive relationship between number of individuals per species and its cultural importance. We recommended including native species truck in conservation strategies.
\end{abstract}

Key words: Colombian Caribbean, rural communities, ethnobotany, mangroves.

Resumen: Jiménez-Escobar, N. D., U. P. Albuquerque \& J. O. Rangel-Ch. 2011. Huertos familiares en la bahía de Cispatá, Córdoba, Colombia. Bonplandia 20(2): 309-328.

Los estudios etnobotánicos relacionados con las comunidades que habitan los alrededores de las zonas estuarinas y aprovechan los bosques de mangle en Colombia son escasos. Por esto, con el fin de apoyar futuros proyectos de manejo y conservación se realizó un primer acercamiento al conocimiento de los huertos familiares de las comunidades campesinas que habitan en la bahía de Cispatá en el Caribe colombiano. Se analizó la estructura, la composición florística y los usos de las especies leñosas presentes en los huertos familiares. En un área total de $29950 \mathrm{~m}^{2}$, correspondiente a doce huertos familiares, se registraron 1798 individuos (DAP $\geq 2,5 \mathrm{~cm}$ ), correspondientes a 83 especies de árboles y palmas, asociadas a 32 familias botánicas. La familia Leguminosae s. I. presentó el mayor número de especies (12), seguida de Annonaceae, Arecaceae y Moraceae (6). La categoría que presentó mayor número de especies fue Comestible (46), seguida de Medicinal (34) y Construcción (33). Según el índice de Saliencia la palmera Cocos nucifera es la especie más importante en los huertos. Se encontró una relación positiva entre el número de individuos por especie y su importancia cultural. Por otra parte, se recomienda incluir en futuros planes de conservación a las especies nativas que presentan un manejo dentro de los huertos.

Palabras clave: Caribe colombiano, comunidades campesinas, etnobotánica, manglares.

${ }^{1}$ Posgrado en Ciencias-Biología. Biodiversidad y Conservación. Instituto de Ciencias Naturales, Facultad de Ciencias, Universidad Nacional de Colombia, Apartado 7495, Bogotá D.C., Colombia, ndjimeneze@unal.edu.co

${ }^{2}$ Laboratorio de Etnobotánica Aplicada (LEA), Departamento de Biología, Área de Botánica, Universidad Federal Rural de Pernambuco, Recife, Brasil, upa@db.ufrpe.br

${ }^{3}$ Instituto de Ciencias Naturales (ICN), Facultad de Ciencias, Universidad Nacional de Colombia, Apartado 7495, Bogotá D.C., Colombia, jorangelc@unal.edu.co 


\section{Introducción}

En las últimas décadas la etnobotánica ha crecido significativamente como disciplina y se ha ampliado más allá de la documentación de usos tradicionales, con transformaciones continuas en su objeto y en sus métodos de abordaje (Phillips, 1996). Esta ciencia ha acumulado una considerable información sobre los recursos y el uso de plantas en los bosques tropicales (Albuquerque \& al., 2005). Sin embargo, se ha ignorado en gran medida a los inmigrantes recientes de las áreas forestales bajo el supuesto de que su conocimiento del bosque es menor y menos interesante que el de las comunidades indígenas (Toledo, 1993; Lawrence \& al., 2005). En Colombia los estudios han sido tradicionalmente enfocados en poblaciones indígenas (véanse: Ortiz, 1989; Sánchez \& Miraña, 1991; Sánchez \& al., 2007; Frausin \& al. 2008; Trujillo-C. \& Correa-Munera, 2010). Sin tener en cuenta que, las comunidades rurales y campesinas, juegan un papel activo en el mantenimiento de los recursos ecológicos y biológicos, y están ligadas directamente a la oferta ambiental, con lo cual suplen muchas de sus necesidades básicas y culturales (Toledo, 1993; Galeano, 2000; Cruz \& al., 2009; Jiménez-Escobar \& al., 2009; Jiménez-Escobar \& EstupiñánGonzález, 2011). En un país como Colombia, donde la población rural es cercana a los diez millones de habitantes (DANE, 2005), los datos cualitativos y cuantitativos sobre el uso de recursos naturales asociados con estas comunidades son fundamentales para la conservación y manejo de los mismos.

Los huertos familiares son sistemas agroforestales asociados a las casas que contribuyen al mantenimiento de importantes funciones ecológicas, económicas y sociales en las comunidades, proporcionando bienestar a millones de familias (FAO, 2000; Pulido $\&$ al., 2008). Producen alimentos para el autoconsumo, derivados agroforestales, además de ingresos económicos adicionales. Se cultivan plantas con fines dendroenergéticos, medicinales, ornamentales, entre otros (FAO, 2000). Recientemente para Latinoamérica, se han realizado trabajos que describen la estructura, la composición y la importancia de las especies vegetales en estos sistemas agroforestales (Álvarez-Bulla \& al., 1989; Padoch \& De Jong, 1991; Rico-Gray \& al., 1991; Rendón \& al., 1998; Lamont \& al., 1999; Wenzel \& Bender, 2003; Albuquerque \& al., 2005; Florentino \& al., 2007; Amorozo \& al., 2008; Pulido \& al., 2008; Fraser \& al., 2011).

La bahía de Cispatá se encuentra ubicada en el departamento de Córdoba, en el litoral colombiano. Presenta vegetación de manglar característica del Caribe Latinoamericano (Hernandez \& Sanchez, 1992; Cortés-Castillo, 2010; Cortés-Castillo \& Rangel-Ch., 2011) con especies como "mangle colorado" (Rhizophora mangle L.), "mangle bobo" (Laguncularia racemosa (L.) C.F. Gaerth), "mangle humo" (Avicennia germinans (L.)L.), "mangle piñuelo" (Pelliciera rizhophorae Planch \& Triana) y "mangle Zaragoza" (Conocarpus erecta L.). Aunque, se conoce la estructura y composición de la flora de esta región (CortésCastillo \& Rangel-Ch., 2011), en la actualidad no hay trabajos que documenten las relaciones entre las comunidades asentadas en la zona y la vegetación en la bahía.

En esta contribución se trata de mostrar el conocimiento y las interacciones de las comunidades rurales asentadas en bahía Cispatá con sus huertos familiares. Se pretende responder las siguientes preguntas: ¿Cómo están organizados y cuál es la composición de especies leñosas en los huertos? ¿Cuáles especies leñosas son consideradas como las más importantes en la comunidad? ¿Hay diferencias entre las especies más importantes según el género de los informantes (hombres y mujeres)? ¿Cuáles son las especies nativas que presentan un manejo en estas áreas?

\section{Materiales y métodos}

\section{Área de estudio}

La bahía de Cispatá, se localiza en el antiguo delta del río Sinú, en el extremo sureste del Golfo de Morrosquillo, en los municipios de San Antero y San Bernardo del Viento, en el departamento de Córdoba (Fig. 1). La región presenta una temperatura 
promedio de $30^{\circ} \mathrm{C}$, una humedad relativa del $80 \%$ y un patrón de distribución de las lluvias de tipo unimodal-biestacional, con montos anuales de $1337 \mathrm{~mm}$ y un promedio mensual de $111 \mathrm{~mm}$, siendo el periodo lluvioso de mayo a noviembre (Rangel-Ch. \& ArellanoPeña, 2010). Según el último censo nacional el número de habitantes del Municipio es de 31455 , donde el $74 \%$ de ellos viven en áreas rurales (DANE, 2005).

El estudio se realizó en el corregimiento de Caño Grande, localizado a $9^{\circ} 23^{`} 51.8^{\prime \prime} \mathrm{N}$ y $75^{\circ}$ $53^{\prime} 44.0^{\prime \prime} \mathrm{W}$, entre 0 y $30 \mathrm{~m}$ de altitud, en el municipio de San Bernardo del Viento, cuenca baja del río Sinú. Este lugar se caracteriza por estar en una zona déltaica, compuesta de múltiples caños (canal), ciénagas y terrazas aluviales. Es una zona de transición entre ecosistemas boscosos de estuarios en buen estado de conservación y bosques secos intervenidos donde predominan especies como Bursera simaruba (L.) Sarg., Ceiba pentandra (L.) Gaertn, Sabal mauritiiformis (H. Karst.) Griseb. \& H. Wendl. y Sterculia apetala (Jacq.) H. Karst. La vegetación natural según Cortés-Castillo \& Rangel-Ch. (2011) está dominada por bosques de mangle y bosques inundables (estuarinos), asociados al gradiente de salinidad y representados por tres tipos de alianzas fitosociológicas: Fico dendrocidae-Rhizphoretum manglis Cortés-Castillo \& Rangel-Ch., Lagunculario
racemosae-Rhizophorion manglis Peinado \& al. y Rhizophorion occidentalis Cuatrecasas.

Para llegar al corregimiento de Caño Grande, se puede acceder por vía terrestre desde el casco urbano del municipio de San Bernardo del Viento (25 minutos en moto), o por vía marítima desde el municipio de San Antero (1 hora en lancha de motor). Todos los habitantes hablan castellano y se definen a sí mismos como una comunidad campesina de agricultores y pescadores. En cuanto a religión, los pobladores están divididos, entre evangélicos y católicos. La comunidad tiene una escuela, donde se imparte la educación básica primaria $\left(1^{\circ}\right.$ a $5^{\circ}$ grado) y la educación básica secundaria $\left(6^{\circ}\right.$ a $9^{\circ}$ grado). En la zona también se identificaron dos asociaciones comunitarias. La primera, es la Asociación de Productores Agrícolas de Caño Grande (APRACAG) y la segunda, es la Asociación de Productores para el Desarrollo Comunitario de la Ciénaga Grande del Bajo Sinú (ASPROCIG).

Hay una clara vocación campesina en los pobladores, dirigida especialmente a la producción de alimento y representado en una tradición de cultivo y manejo de especies en los huertos (Fals-Borda 1986). Se cultivan principalmente especies alimentarias como "arroz" (Oryza sativa L.), "banano" (Musa x paradisiaca L.), "coco" (Cocos nucifera L.) y en menor proporción "mango" (Mangifera indica

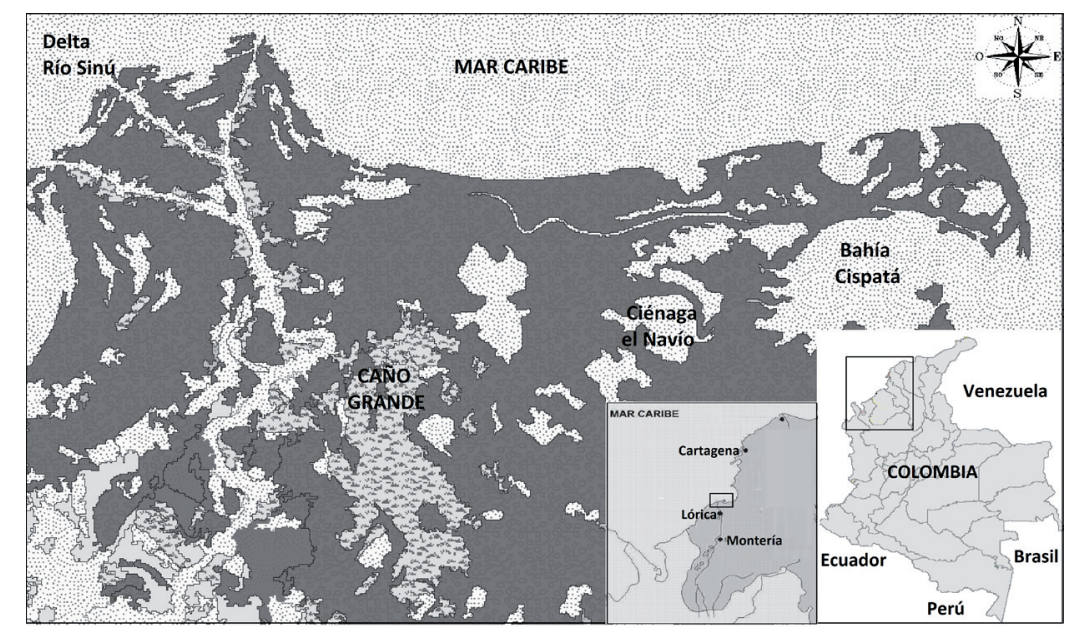

Fig. 1. Localización del área de estudio, vereda Caño Grande, Municipio de San Bernardo del Viento, Departamento de Córdoba, Caribe colombiano. (Modificado de Cortés-Castillo 2010). 
L.), "ñame" (Dioscorea alata L.) y "papaya" (Carica papaya L.). Es una economía de subsistencia, con pequeños excedentes que se reúnen para comercializar en los municipios de Lórica y Montería (departamento de Córdoba). Los hombres principalmente se dedican a la pesca con las técnicas de trasmallo y atarraya, tanto en mar abierto como en ciénagas de agua dulce y manglares. Aunque, esta actividad genera recursos económicos para las familias, gran parte del pescado es consumido en la región. La actividad ganadera es muy baja y está orientada a la cría de ganado vacuno para autoconsumo. En la actualidad en la bahía hay un recrudecimiento del conflicto armado generado por grupos al margen de la ley, que buscan el control de la zona, clave en los negocios ilícitos de alucinógenos.

\section{Fase de campo}

Se realizaron tres salidas de campo a la comunidad de Caño Grande entre marzo de 2010 y enero de 2011. Los datos etnobotánicos y socioeconomicos se obtuvieron por medio de entrevistas semiestructuradas siguiendo el modelo propuesto por Jiménez-Escobar \& al. (2009) con algunas modificaciones. Se indagó principalmente sobre las formas de uso y los nombres comunes de los árboles útiles de la región, tanto en áreas naturales como en huertos familiares.

Participaron 35 personas pertenecientes a diferentes familias (16 mujeres y 19 hombres), cuyas edades variaron entre los 19 y 68 años de edad. Adicionalmente, se realizaron entrevistas informales y caminatas guiadas -con preguntas espontáneas que fueron surgiendo en el transcurso de la conversaciónfacilitando la comunicación y comprensión de los temas a tratar (Karremans, 1994; Albuquerque \& al., 2010).

Para el sector del corregimiento de Caño Grande más próximo a las ciénagas de agua dulce, se identificaron cerca de 40 huertos familiares, de éstos fueron seleccionados al azar doce. Con el consentimiento previo de su dueño, se midió el área total de cada huerto $\left(\mathrm{m}^{2}\right)$ y se elaboró un inventario de las especies de árboles o palmas con un diámetro a la altura del pecho (DAP) superior a $2,5 \mathrm{~cm}$. Se estimó para cada individuo la altura $(\mathrm{m})$, la cobertura $\left(\mathrm{m}^{2}\right)$ y el DAP $(\mathrm{cm})$, como lo sugiere RangelCh. \& Velázquez (1997).

Con base en las categorías y consideraciones empleadas por varios investigadores colombianos (Galeano, 2000; Orjuela-R. \& al., 2004; Marin-Corba \& al., 2005; Cruz \& al., 2009; Jiménez-Escobar \& al., 2009; Estupiñán-González \& Jiménez-Escobar, 2010; Trujillo-C. \& Correa-Munera, 2010; Estupiñán-González \& al., 2011; JiménezEscobar \& Estupiñán-González, 2011), se definieron doce categorías de uso, lo cual permitió facilitar las comparaciones entre diferentes estudios realizados en Colombia. La clasificación de categorías de uso se realizó de la siguiente forma: Agropecuaria (Ag): abono, forraje, insecticidas y veterinario; Artesanal (Ar): accesorios, instrumentos musicales, tallas en madera y vestidos; Cercas vivas $(\mathrm{Cv})$; Comestible (Co): aceites, bebidas, bebidas alcohólicas, condimentos, dulces, frutales, galletas, semillas tostadas y vegetales; Construcción (C): ebanistería, cercas, canoas, maderable y no maderable; Leña (Lñ); Lúdica (Lu); Mágico-religiosa (M$\mathrm{R}$ ); Medicinal (Me); Servicios Ambientales (Sa); Ornamental (Or); Tecnológica (Tc): cosmético, herramientas y pegantes. Aunque, los análisis se realizaron a nivel de categorías, información más detallada acerca de la forma de uso se incluye en la Tabla 1.

Se realizó recolecta de material botánico en compañía de algunos de los habitantes reconocidos como los mayores conocedores de plantas de la zona. Toda la colección fue preservada según los estándares establecidos, los especímenes fueron determinados y posteriormente depositados en el Herbario Nacional Colombiano (COL), del Instituto de Ciencias Naturales de la Universidad Nacional de Colombia en Bogotá, bajo la serie N.D. Jiménez-Escobar. La nomenclatura taxonómica sigue la usada en W3Tropicos (Missouri Botanical Garden VAST-VAScular Tropicos) y confirmada con The Plant List (2010).

\section{Análisis de datos}

La abundancia absoluta corresponde al 
número de individuos de cada una de las especies encontradas en los huertos. La frecuencia relativa corresponde al número de huertos donde se encontró la especie dividida por el total de huertos medidos. El origen geográfico de las especies (nativas o introducidas) encontradas en los huertos fue evaluado en relación a los bosques estuarinos y de manglar de la bahía de Cispatá (Cortés-Castillo, 2010; Cortés-Castillo \& Rangel-Ch., 2011; Cortés-Castillo \& RangelCh., En imprenta) y complementado con la información de bosques secos (Rivera-Díaz, 2010) y de bosques húmedos (EstupiñánGonzález \& al., 2011) del departamento de Córdoba. Las especies con area de distribución geográfica natural en el Caribe colombiano, se consideraron como nativas.

Para el análisis de parámetros de la estructura de los huertos se realizaron diagramas con clases de distribución de altura, de cobertura y de DAP, con base en la ley de Sturges (Rangel-Ch. \& Velásquez, 1997), de la siguiente manera: $\mathrm{C}=(\mathrm{X}$ máx-Xmín) $/ \mathrm{m}$, donde: $m=1+3,3 x(\log n), n=$ número total de individuos, $\mathrm{c}=$ amplitud del intervalo $\mathrm{y}$ $\mathrm{x}=$ parámetro a analizar.

Se realizaron asociaciones entre el tamaño de la huerta, número de especies y el número de individuos, utilizando el coeficiente de correlación de Spearman (Sokal \& Rolf, 1995). Para establecer la importancia de las especies nativas en los huertos, se realizó una prueba de Chi-cuadrado (proporciones esperadas iguales), para la abundancia y la riqueza de las especies según su origen geográfico, empleando el programa Bioestat 5.0 (Ayres \& al. 2007).

Con el fin de establecer cuáles son las especies que la comunidad considera más importantes, al final de cada entrevista se enfatizó en una última pregunta: ¿De todas las especies que el señor(a) mencionó, cuáles son las tres más importantes? es más, si solo pudiera tener en su huerto tres especies ¿Cuáles tendría y por qué? Con las respuestas se realizó un análisis de frecuencia y posición de las especies nombradas por cada entrevistado: este índice se denomina de Saliencia cultural y fue calculado empleando el programa Anthropac 4.0 (Borgatii \& Natick, 1996).
Para establecer la relación entre las especies más importantes y su representatividad en los huertos se realizó una regresión lineal simple comparando la Saliencia cultural con la abundancia.

\section{Resultados y Discusión}

\section{Composición de los huertos familiares}

En un área muestreada de $29950 \mathrm{~m}^{2}$, que incluye doce huertos familiares se registraron 83 especies de árboles y palmas, que corresponden a 61 géneros y 32 familias (Tabla 1). La familia Leguminosae sensu lato presentó el mayor número de especies (12), seguida de Annonaceae, Arecaceae y Moraceae (6), y Anacardiaceae, Malvaceae y Meliaceae (4). Según Pulido \& al. (2008) la riqueza de los huertos en Latinoamérica es muy variable, desde 27 a 405 especies, incluyendo hierbas y bejucos. Si se comparan los resultados obtenidos sobre la riqueza de árboles y palmas en huertos de zonas secas del continente, los valores registrados en la bahía de Cispatá son altos. En la Caatinga brasilera se han registrado entre 36-46 especies (Albuquerque \& al., 2005; Florentino \& al., 2007), mientras que para tierras bajas y semiáridas en Cuba 23 especies (Wezel \& Bender, 2003). No se encontraron trabajos comparables para bosques de mangle o bosques estuarinos en Latinoamérica.

Cuatro especies están presentes en todos los huertos familiares: Annona muricata, Cocos nucifera, Mangifera indica y Tabebuia rosea, las tres primeras corresponden a especies comestibles, que hacen parte de la dieta habitual de los pobladores y que además son comercializadas y $T$. rosea, es una de las especies maderables más conocidas de la región (Jiménez-Escobar \& Estupiñán-González, 2011). Otras especies con una frecuencia relativa alta son: Cedrela odorata, Crescentia cujete y Psidium guajava (91,7), Bixa orellana, Carica papaya y Citrus reticulata $(83,3)$.

Al nivel de abundancia en todos los huertos, la especie con más individuos es C. nucifera (302), seguida de $T$. rosea (223), S. mauritiiformis (130), Sapium glandulosum (112), Gliricidia sepium (102) y C. odorata (101). Estas seis 
especies concentran $53 \%$ de los valores de la abundanciaen los huertos. C. nucifera es la única especie comestible. La hoja de la "palma amarga" ( $S$. mauritiformis) es considerada entre los habitantes de la región, el material preferido para techar, mientras las especies restantes corresponden a árboles maderables o utilizados como dendroenergéticos, que hacen parte de las cercas vivas que delimitan las propiedades.

Según la caracterización florística y estructural de la vegetación de la bahía de Cispatá (Cortés-Castillo, 2010; Cortés-Castillo \& Rangel-Ch., 2011), de las 83 especies registradas para los huertos familiares, nueve se encuentran en áreas boscosas naturales como en riberas de caños: Coccoloba acuminata, en pantanos: Annona glabra, Bactris guineensis, C. cujete, Ficus dendrocida y Albizia saman, en bosques de mangle: Conocarpus erecta y Maclura tictorea, y en bosques de estuario y de manglar $G$. sepium y $T$. rosea. Estas nueve especies son usadas por los habitantes de la comunidad, su presencia en los huertos puede ser atribuida a que toleran niveles altos de salinidad, lo cual permite que se establezcan en los huertos con facilidad y sin requerir de muchos cuidados. De éstas, $C$. cujete, $C$. erecta, $G$. sepium y $T$. rosea son cultivadas dentro del huerto, las demás crecen naturalmente y no son cortadas. Todas ellas son especies tradicionalmente conocidas $\mathrm{y}$ usadas por otras comunidades campesinas en el Caribe colombiano (Jiménez-Escobar \& Estupiñán-González, 2011). Sumadas a otras 34 especies consideradas como nativas para el departamento de Córdoba, completan 43 especies, que contabilizan 975 individuos, siendo las más frecuentes en los huertos T. rosea, C. odorata y C. cujete. De las 40 especies restantes, 39 corresponden a las introducidas, con 822 individuos, de las cuales las más frecuentes son: $C$. nucifera y $M$. indica. Según los datos de origen geográfico, la abundancia de especies nativas en los huertos muestra un dominio marcado $\left(\mathrm{x}^{2}=\right.$ 13,027; $p=0,003)$. En el caso de la riqueza, la diferencia de proporciones entre las especies nativas y las introducidas no es significativa $\left(x^{2}=0,195 ; p=0,7404\right)$. Estos resultados son contrarios a los encontrados para otros trabajos en regiones tropicales, donde la riqueza y la abundancia de las especies introducidas es superior (Rico-Gray \& al., 1991; Albuquerque \& al., 2005; Florentino \& al., 2007). Los valores obtenidos para la riqueza y abundancia de las nativas en Cispatá pueden ser atribuidos a especies como: Elaeis oleifera, Manilkara zapota (L.) Van Royen, Pouteria sapota (Jacq.) H. E. Moore \& Steam, Sabal mauritiiformis y Swietenia macrophylla King., que son consideradas nativas de la región Caribe. No crecen en el interior de los bosques estuarinos o de manglar de la bahía, pero si lo hacen en localidades cercanas; su importancia cultural y reconocimiento local hacen que los pobladores las incluyan en de los huertos.

Los huertos familiares tropicales de todo el mundo incluyen actualmente un grupo de especies arbóreas como: Citrus spp., C. nucifera, Coffea arabica, $M$. indica, con especies nativas de América como: Carica papaya, G. sepium, Leucaena leucocephala y Psidium guajava (Pulido \& al., 2008). Con excepción de $C$. arabica y L. leucocephala, todas se encuentran presentes en los huertos familiares de la bahía de Cispatá. Otras especies comunes que se encontraron en la región y que están presentes en otros huertos latinoamericanos son: Annona muricata, Persea americana y Spondias mombin (Álvarez-Bulla \& al., 1989; Padoch \& De Jong, 1991; Rico-Gray \& al., 1991; Rendon \& al., 1998; Lamont \& al., 1999; Wenzel \& Bender, 2003; Albuquerque \& al., 2005; Florentino \& al., 2007).

Es importante resaltar que los cultivos de "arroz" (O. sativa) y "banano" (M. x paradisiaca) presentes dentro de los huertos familiares hacen parte de la dieta básica de los pobladores y además son comercializados. El "arroz" es sembrado a las orillas del "caño" (en contacto directo con el agua) y posteriormente transplantado a tierras menos húmedas, las infrutescencias son colectadas de forma manual y luego se ponen a secar en los techos de las viviendas. También, de forma manual y con ayuda de un pilón (mortero de hasta $1 \mathrm{~m}$ de altura) se descascara el fruto -eliminando el pericarpo- para consumo local. Cuando éste va a ser vendido, la cosecha es llevada a la única trilladora de la región, donde comienza su proceso de comercialización. El "banano" 
N. D. Jiménez-Escobar \& al., Huertos familiares en la bahía de Cispatá, Córdoba, Colombia

Tabla 1. Lista comentada de las especies leñosas encontradas en los huertos familiares en la bahía de Cispatá, Córdoba, Colombia.

\begin{tabular}{|c|c|c|c|c|c|c|}
\hline Especies & Nombre común & Ind. & $\begin{array}{c}\text { Fr. } \\
\mathrm{n}=12\end{array}$ & origen & $\begin{array}{l}\text { Parte } \\
\text { usada }\end{array}$ & Uso \\
\hline \multicolumn{7}{|l|}{ ANACARDIACEAE } \\
\hline Anacardium occidentale L. & marañón & $1(0,06 \%)$ & 8,33 & I & Fr, Se & Co-f, Co-st \\
\hline Mangifera indica L. & mango & $63(3,50 \%)$ & 100,00 & I & $\begin{array}{l}\mathrm{Cz}, \mathrm{Fr}, \mathrm{Pl} \\
\mathrm{Tr}\end{array}$ & $\begin{array}{l}\text { Ag-fe, Cv, Co-f, } \\
\text { Co-b, Co-d, } \\
\text { C-m,C-c, Lñ, Me, } \\
\text { Or, Te-he }\end{array}$ \\
\hline Spondias mombin L. & jobo & $6(0,33 \%)$ & 25,00 & $\mathrm{~N}$ & Fr, Pl, Tr & $\begin{array}{l}\mathrm{Ar}, \mathrm{Cv}, \mathrm{Co}-\mathrm{f} \\
\mathrm{C}-\mathrm{m}, \mathrm{C}-\mathrm{e}, \mathrm{C}-\mathrm{c}\end{array}$ \\
\hline Spondias purpurea L. & $\begin{array}{l}\text { ciruelo, ciruela } \\
\text { (fruto) }\end{array}$ & $17(0,95 \%)$ & 33,33 & I & Fr, $\mathrm{Pl}$ & $\mathrm{Cv}, \mathrm{Co}-\mathrm{f}$ \\
\hline \multicolumn{7}{|l|}{ ANNONACEAE } \\
\hline Annona cherimola Mill. & chirimoya & $3(0,17 \%)$ & 25,00 & I & Fr, & Co-f \\
\hline Annona glabra L. & $\begin{array}{l}\text { guanabanitaepuy, } \\
\text { maguey }\end{array}$ & $14(0,78 \%)$ & 33,33 & $\mathrm{~N}^{*}$ & Fr, Pl, Tr & $\begin{array}{l}\text { Cv, Co-f, Co-b, } \\
\text { C-m,C-va, C-e, } \\
\text { C-c, Lñ }\end{array}$ \\
\hline Annona muricata L. & guanábano & $76(4,23 \%)$ & 100,00 & I & $\mathrm{Cg}, \mathrm{Fr}, \mathrm{Hj}$ & $\begin{array}{l}\text { Co-f, Co-b, Co-d, } \\
\mathrm{Me}\end{array}$ \\
\hline $\begin{array}{l}\text { Annona purpurea Moc. \& } \\
\text { Sessé ex Dunal }\end{array}$ & guanacona & $1(0,06 \%)$ & 8,33 & I & $\mathrm{Hj}, \mathrm{Tr}$ & $\mathrm{M}-\mathrm{R}, \mathrm{Me}$ \\
\hline Annona squamosa $\mathrm{L}$. & anón & $6(0,33 \%)$ & 25,00 & I & $\mathrm{Fr}$ & Co-f \\
\hline $\begin{array}{l}\text { Cananga odorata (Lam.) } \\
\text { Hook.f. \& Thomson }\end{array}$ & flor de amor & $2(0,11 \%)$ & 16,67 & I & $\mathrm{Fl}, \mathrm{Hj}, \mathrm{Pl}$ & M-R, Me, Or \\
\hline \multicolumn{7}{|l|}{ APOCYNACEAE } \\
\hline $\begin{array}{l}\text { Cascabela thevetia (L.) } \\
\text { Lippold }\end{array}$ & calabonga & $2(0,11 \%)$ & 16,67 & I & $\mathrm{Pl}$ & M-R \\
\hline \multicolumn{7}{|l|}{ ARECACEAE } \\
\hline $\begin{array}{l}\text { Bactris guineensis (L.) H.E. } \\
\text { Moore }\end{array}$ & $\begin{array}{l}\text { lata, lata } \\
\text { espinosa, } \\
\text { corozo(fruto) }\end{array}$ & $1(0,06 \%)$ & 8,33 & $N^{*}$ & Fr, $\operatorname{Tr}$ & $\begin{array}{l}\text { Ar, Co-b, Co-ba, } \\
\text { Co-d, C-m,C-c }\end{array}$ \\
\hline Cocos nucifera $\mathrm{L}$. & $\begin{array}{l}\text { palma de coco, } \\
\text { coco(fruto) }\end{array}$ & $\begin{array}{c}302 \\
(16,80 \%)\end{array}$ & 100,00 & I & $\begin{array}{l}\mathrm{Fr}, \mathrm{Hj}, \mathrm{Pl} \\
\mathrm{Se}, \mathrm{Tr}\end{array}$ & $\begin{array}{l}\text { Ar, Co-f, Co-b, } \\
\text { Co-a, Co-g, Co-d, } \\
\text { C-m,C-c, Lñ, Me, } \\
\text { Te-he }\end{array}$ \\
\hline $\begin{array}{l}\text { Elaeis oleifera (Kunth) } \\
\text { Cortés }\end{array}$ & $\begin{array}{l}\text { palma de corozo, } \\
\text { coroza(fruto) }\end{array}$ & $31(1,72 \%)$ & 58,33 & $\mathrm{~N}$ & $\begin{array}{l}\mathrm{Cg}, \mathrm{Fr}, \mathrm{Hj} \\
\mathrm{Pl}, \mathrm{Tr}\end{array}$ & $\begin{array}{l}\text { Ag-fe, Ar, Co-f, } \\
\text { Co-b, Co-ba, Co-a, } \\
\text { C-m,C-c, Me, Or, } \\
\text { Te-co, Te-he }\end{array}$ \\
\hline $\begin{array}{l}\text { Livistona chinensis (Jacq.)R. } \\
\text { Br. Ex Mart. }\end{array}$ & palma de lujo & $4(0,22 \%)$ & 25,00 & I & $\mathrm{Pl}$ & Or \\
\hline $\begin{array}{l}\text { Sabal mauritiiformis (H. } \\
\text { Karst.) Griseb. \& H. Wendl. }\end{array}$ & palma amarga & $\begin{array}{c}130 \\
(7,23 \%)\end{array}$ & 58,33 & $\mathrm{~N}$ & $\mathrm{Hj}, \mathrm{Pl}$ & Or, Te-he \\
\hline $\begin{array}{l}\text { Veitchia merrillii (Becc.) } \\
\text { H.E. Moore }\end{array}$ & palma de lujo & $2(0,11 \%)$ & 16,67 & I & $\mathrm{Pl}$ & Or \\
\hline \multicolumn{7}{|l|}{ BIGNONIACEAE } \\
\hline Crescentia cujete L. & totumo & $24(1,33 \%)$ & 91,67 & $\mathrm{~N}^{*}$ & $\begin{array}{l}\text { Fl, Fr, Pl, } \\
\text { Tr }\end{array}$ & $\begin{array}{l}\text { Ag-fe, Ag-v, Ar, } \\
\text { Cv, C-m,Lu, Me, } \\
\text { Or, Te-co, Te-he }\end{array}$ \\
\hline
\end{tabular}




\begin{tabular}{|c|c|c|c|c|c|c|}
\hline Especies & Nombre común & Ind. & $\underset{n=12}{\text { Fr. }}$ & origen & $\begin{array}{l}\text { Parte } \\
\text { usada }\end{array}$ & Uso \\
\hline $\begin{array}{l}\text { Tabebuia rosea (Bertol) A. } \\
\text { DC. }\end{array}$ & roble & $\begin{array}{c}223 \\
(12,40 \%)\end{array}$ & 100,00 & $\mathrm{~N}^{*}$ & $\begin{array}{l}\mathrm{Cz}, \mathrm{Hj}, \mathrm{Pl} \\
\mathrm{Se}, \mathrm{Tr}\end{array}$ & $\begin{array}{l}\text { Ar, Cv, C-m,C-va, } \\
\text { C-e, Lñ, Lu, Me, } \\
\text { Te-he }\end{array}$ \\
\hline \multicolumn{7}{|l|}{ BIXACEAE } \\
\hline Bixa orellana $\mathrm{L}$. & achiote & $29(1,61 \%)$ & 83,33 & I & $\mathrm{Cz}, \mathrm{Hj}, \mathrm{Pl}$ & $\mathrm{Co}-\mathrm{c}, \mathrm{Me}, \mathrm{Or}$ \\
\hline \multicolumn{7}{|l|}{ BORAGINACEAE } \\
\hline Cordia dentata Poir. & asauco, uvito & $3(0,17 \%)$ & 8,33 & $\mathrm{~N}$ & $\begin{array}{l}\mathrm{Cz}, \mathrm{Ex}, \mathrm{Fl}, \\
\mathrm{Fr}, \mathrm{Pl}, \mathrm{Se}, \\
\mathrm{Tr}\end{array}$ & $\begin{array}{l}\text { Cv, Co-f, C-m,Me, } \\
\text { Te-co, Te-pe, Te-pc }\end{array}$ \\
\hline Cordia aff. nodosa Lam. & muñeco & $1(0,06 \%)$ & 8,33 & $\mathrm{~N}$ & $\mathrm{Fr}, \mathrm{Pl}, \mathrm{Tr}$ & $\begin{array}{l}\text { Ag-fe, Cv, Co-f, } \\
\text { C-m }\end{array}$ \\
\hline \multicolumn{7}{|l|}{ BURSERACEAE } \\
\hline $\begin{array}{l}\text { Bursera graveolens (Kunth) } \\
\text { Triana \& Planch. }\end{array}$ & caraña & $4(0,22 \%)$ & 8,33 & $\mathrm{~N}$ & $\mathrm{Ex}, \mathrm{Fl}, \mathrm{Hj}$ & Me, Te-pe \\
\hline Bursera simaruba (L.) Sarg. & indio en cueros & $19(1,06 \%)$ & 41,67 & $\mathrm{~N}$ & $\mathrm{Pl}, \mathrm{Tr}$ & $\mathrm{Cv}, \mathrm{C}-\mathrm{m}$ \\
\hline \multicolumn{7}{|l|}{ CAPPARACEAE } \\
\hline Capparis odoratissima Jaqc. & olivo & $3(0,17 \%)$ & 8,33 & $\mathrm{~N}$ & $\mathrm{Hj}, \mathrm{Pl}$ & $\mathrm{M}-\mathrm{R}, \mathrm{Me}$ \\
\hline Crateva tapia $\mathrm{L}$. & cachimondo & $1(0,06 \%)$ & 8,33 & $\mathrm{~N}$ & $\mathrm{Pl}, \mathrm{Tr}$ & $\begin{array}{l}\text { Cv, C-m,C-c, Lñ, } \\
\text { Te-he }\end{array}$ \\
\hline \multicolumn{7}{|l|}{ CARICACEAE } \\
\hline Carica papaya $\mathrm{L}$. & papayo & $65(3,62 \%)$ & 83,33 & I & $\mathrm{Ex}, \mathrm{Fr}$ & $\begin{array}{l}\text { Co-f, Co-b, Co-d, } \\
\text { Me, As-f, Te-co, } \\
\text { Te-pe }\end{array}$ \\
\hline \multicolumn{7}{|l|}{ CECROPIACEAE } \\
\hline Cecropia cf. peltata L. & guarumo & $27(1,50 \%)$ & 75,00 & $\mathrm{~N}$ & $\mathrm{Cg}, \mathrm{Hj}, \mathrm{Tr}$ & C-m,Lñ, M-R, Me \\
\hline \multicolumn{7}{|l|}{ COMBRETACEAE } \\
\hline Conocarpus erecta L. & $\begin{array}{l}\text { mangle zaragoza, } \\
\text { zaragoza }\end{array}$ & $9(0,50 \%)$ & 50,00 & $\mathrm{~N}^{*}$ & $\mathrm{Pl}, \mathrm{Tr}$ & $\begin{array}{l}\text { Cv, C-m,C-c, Lñ, } \\
\text { Or, Te-he }\end{array}$ \\
\hline \multicolumn{7}{|l|}{ EUPHORBIACEAE } \\
\hline Jathropha sp. 1 & cereza & $1(0,06 \%)$ & 8,33 & I & $\mathrm{Fr}$ & $\begin{array}{l}\text { Co-f, Co-b, Co-d, } \\
\text { As-f }\end{array}$ \\
\hline Ricinus comunis L. & higuereta & $2(0,11 \%)$ & 16,67 & I & $\mathrm{Hj}$ & $\mathrm{Me}$ \\
\hline $\begin{array}{l}\text { Sapium glandulosum (L.) } \\
\text { Morong }\end{array}$ & ñipi & $\begin{array}{c}112 \\
(6,23 \%)\end{array}$ & 66,67 & $\mathrm{~N}$ & $\mathrm{Ex}, \mathrm{Pl}, \mathrm{Tr}$ & $\begin{array}{l}\text { Cv, C-m,C-c, Lñ, } \\
\text { Or, Te-pe, Te-pc }\end{array}$ \\
\hline \multicolumn{7}{|l|}{ LAMIACEAE } \\
\hline Tectona grandis L. f. & teca & $1(0,06 \%)$ & 8,33 & I & $\operatorname{Tr}$ & $\mathrm{C}-\mathrm{m}$ \\
\hline \multicolumn{7}{|l|}{ LAURACEAE } \\
\hline Persea americana Mill. & aguacate & $18(1,00 \%)$ & 58,33 & I & $\begin{array}{l}\mathrm{Cg}, \mathrm{Fr}, \mathrm{Hj} \\
\mathrm{Tr}\end{array}$ & $\begin{array}{l}\text { Co-f, Co-v, Lñ, } \\
\text { Me, Te-co }\end{array}$ \\
\hline \multicolumn{7}{|l|}{ LEGUMINOSAE } \\
\hline Albizia saman (Jacq.) Merr. & campano & $6(0,33 \%)$ & 16,67 & $N^{*}$ & $\mathrm{Fr}, \mathrm{Pl}, \mathrm{Tr}$ & $\begin{array}{l}\text { Ag-fe, Co-d, } \\
\text { C-m,C-va, C-e, } \\
\text { C-c, Or, Te-he }\end{array}$ \\
\hline $\begin{array}{l}\text { Caesalpinia pulcherrima } \\
\text { L. Sw. }\end{array}$ & flor de muerto & $2(0,11 \%)$ & 16,67 & I & $\mathrm{Pl}$ & Or \\
\hline Cassia grandis L. f. & caña fistula & $1(0,06 \%)$ & 8,33 & $\mathrm{~N}$ & Fr, $\mathrm{Hj}$ & $\mathrm{Co}-\mathrm{b}, \mathrm{Me}$ \\
\hline
\end{tabular}


N. D. Jiménez-Escobar \& al., Huertos familiares en la bahía de Cispatá, Córdoba, Colombia

\begin{tabular}{|c|c|c|c|c|c|c|}
\hline Especies & Nombre común & Ind. & $\begin{array}{c}\mathrm{Fr} . \\
\mathrm{n}=12\end{array}$ & origen & $\begin{array}{l}\text { Parte } \\
\text { usada }\end{array}$ & Uso \\
\hline $\begin{array}{l}\text { Delonix regia (Bojer nex } \\
\text { Hook.) Raf. }\end{array}$ & acacio & $2(0,11 \%)$ & 16,67 & $\mathrm{I}$ & $\mathrm{Pl}$ & Or \\
\hline $\begin{array}{l}\text { Enterolobium cyclocarpum } \\
\text { (Jacq.) Griseb. }\end{array}$ & carito & $2(0,11 \%)$ & 16,67 & $\mathrm{~N}$ & $\operatorname{Tr}$ & $\mathrm{C}-\mathrm{m}, \mathrm{C}-\mathrm{c}$ \\
\hline $\begin{array}{l}\text { Gliricidia sepium (Jacq.) } \\
\text { Kunth ex Walp. }\end{array}$ & matarratón & $\begin{array}{c}102 \\
(5,67 \%)\end{array}$ & 66,67 & $\mathrm{~N}^{*}$ & $\begin{array}{l}\text { Ex, } \mathrm{Hj}, \mathrm{Pl}, \\
\operatorname{Tr}\end{array}$ & $\begin{array}{l}\text { Ag-a, Ag-fe, Ag-i, } \\
\text { Cv, C-m,Lñ, Lu, } \\
\text { Me, Or, Te-he }\end{array}$ \\
\hline Hymenaea courbaril L. & algarrobo & $2(0,11 \%)$ & 8,33 & $\mathrm{~N}$ & $\mathrm{Fr}, \operatorname{Tr}$ & Co-b, C-m \\
\hline Inga edulis Mart. & guamo & $12(0,67 \%)$ & 41,67 & $\mathrm{~N}$ & Fr, Pl, Tr & Cv, Co-f, Lñ \\
\hline Inga sp. 1 & guamo & $4(0,22 \%)$ & 25,00 & $\mathrm{~N}$ & $\mathrm{Fr}$ & Co-f \\
\hline Inga sp. 2 & guama pelua & $2(0,11 \%)$ & 8,33 & $\mathrm{~N}$ & $\operatorname{Tr}$ & Lñ \\
\hline $\begin{array}{l}\text { Pithecellobium lanceolatum } \\
\text { (Humb. \& Bonpl. ex Wild.) } \\
\text { Benth. }\end{array}$ & $\begin{array}{l}\text { carne fresca, } \\
\text { espino }\end{array}$ & $5(0,28 \%)$ & 33,33 & $\mathrm{~N}$ & $\mathrm{Fr}, \mathrm{Pl}, \mathrm{Tr}$ & $\begin{array}{l}\text { Cv, Co-f, C-m,C-c, } \\
\text { Lñ }\end{array}$ \\
\hline Tamarindus indica $\mathrm{L}$. & tamarindo & $4(0,22 \%)$ & 25,00 & I & $\begin{array}{l}\mathrm{Cg}, \mathrm{Fr}, \mathrm{Hj} \\
\mathrm{Pl}, \mathrm{Tr}\end{array}$ & $\begin{array}{l}\text { Co-b, Co-d, } \\
\text { C-m,M-R, Me }\end{array}$ \\
\hline \multicolumn{7}{|l|}{ LYTHRACEAE } \\
\hline Lawsonia inermis $\mathrm{L}$. & reseda & $1(0,06 \%)$ & 8,33 & I & $\mathrm{Hj}, \mathrm{Pl}$ & $\mathrm{Me}$, Or \\
\hline Punica granatum $\mathrm{L}$. & granada & $1(0,06 \%)$ & 8,33 & I & $\mathrm{Fr}, \mathrm{Hj}$ & Co-b, Co-d, Me \\
\hline \multicolumn{7}{|l|}{ MALPIGHIACEAE } \\
\hline Malpighia emarginata DC. & cereza & $6(0,33 \%)$ & 33,33 & $\mathrm{~N}$ & $\mathrm{Fr}$ & $\begin{array}{l}\text { Co-f, Co-b, Co-d, } \\
\text { As-f }\end{array}$ \\
\hline \multicolumn{7}{|l|}{ MALVACEAE } \\
\hline Guazuma ulmifolia L. & guácimo & $40(2,22 \%)$ & 58,33 & $\mathrm{~N}$ & $\begin{array}{l}\mathrm{Cz}, \mathrm{Fr}, \mathrm{Pl} \\
\mathrm{Se}, \mathrm{Tr}\end{array}$ & $\begin{array}{l}\text { Ag-fe, Ar, Cv, } \\
\text { Co-f, C-m,Lñ, Me, } \\
\text { Te-co, Te-he }\end{array}$ \\
\hline $\begin{array}{l}\text { Malvaviscus cf. arboreus } \\
\text { Cav. }\end{array}$ & azuquita & $1(0,06 \%)$ & 8,33 & I & $\mathrm{Hj}$, & $\mathrm{Me}$ \\
\hline $\begin{array}{l}\text { Sterculia apetala (Jacq.) H. } \\
\text { Karst. }\end{array}$ & camajón, tuntún & $3(0,17 \%)$ & 16,67 & $\mathrm{~N}$ & $\mathrm{Cz}, \mathrm{Se}, \mathrm{Tr}$ & Ar, Co-st, Te-he \\
\hline Theobroma cacao $\mathrm{L}$. & cacao & $10(0,56 \%)$ & 50,00 & I & $\mathrm{Fr}$ & Co-f, Co-b, Co-d \\
\hline \multicolumn{7}{|l|}{ MELIACEAE } \\
\hline Azadirachta indica A. Juss. & ni, nim, nui & $4(0,22 \%)$ & 25,00 & I & $\begin{array}{l}\mathrm{Cg}, \mathrm{Cz} \\
\mathrm{Hj}, \mathrm{Tr}\end{array}$ & $\begin{array}{l}\text { Ag-i, C-m,M-R, } \\
\mathrm{Me}\end{array}$ \\
\hline Cedrela odorata $\mathrm{L}$. & cedro & $\begin{array}{c}101 \\
(5,62 \%)\end{array}$ & 91,67 & $\mathrm{~N}$ & $\begin{array}{l}\mathrm{Cz}, \mathrm{Fr}, \mathrm{Hj} \\
\mathrm{Pl}, \mathrm{Se}, \mathrm{Tr}\end{array}$ & $\begin{array}{l}\mathrm{Ar}, \mathrm{Cv}, \mathrm{C}-\mathrm{m}, \mathrm{C}-\mathrm{va}, \\
\mathrm{C}-\mathrm{e}, \mathrm{L} \tilde{n}, \mathrm{Lu}, \mathrm{Me}\end{array}$ \\
\hline Melia azedarach $\mathrm{L}$. & paraíso & $3(0,17 \%)$ & 25,00 & I & $\mathrm{Hj}, \mathrm{Pl}$ & $\mathrm{M}-\mathrm{R}, \mathrm{Me}$ \\
\hline $\begin{array}{l}\text { Swietenia macrophylla } \\
\text { King. } \\
\text { MORACEAE }\end{array}$ & caoba & $2(0,11 \%)$ & 8,33 & $\mathrm{~N}$ & $\operatorname{Tr}$ & $\mathrm{C}-\mathrm{m}$ \\
\hline $\begin{array}{l}\text { Artocarpus altilis } \\
\text { (Parkinson) Fosberg }\end{array}$ & pan de dios & $2(0,11 \%)$ & 16,67 & I & $\begin{array}{l}\text { Ex, Fr, Se, } \\
\operatorname{Tr}\end{array}$ & $\begin{array}{l}\text { Co-b, Co-v, Co-g, } \\
\text { Co-d, Me, Te-he, } \\
\text { Te-pe }\end{array}$ \\
\hline $\begin{array}{l}\text { Artocarpus cf. integrifolia } \\
\text { L.f. }\end{array}$ & árbol pan & $9(0,50 \%)$ & 50,00 & I & $\mathrm{Ex}, \mathrm{Fr}, \mathrm{Tr}$ & $\begin{array}{l}\text { Co-b, Co-v, Co-g, } \\
\text { Co-d, C-m,Te-pe }\end{array}$ \\
\hline Ficus dendrocida Kunth. & higo & $1(0,06 \%)$ & 8,33 & $\mathrm{~N}^{*}$ & $\operatorname{Tr}$ & Lñ \\
\hline Ficus insipida Willd. & higuerón & $1(0,06 \%)$ & 8,33 & $\mathrm{~N}$ & Fl & M-R \\
\hline
\end{tabular}




\begin{tabular}{|c|c|c|c|c|c|c|}
\hline Especies & Nombre común & Ind. & $\begin{array}{c}\text { Fr. } \\
\mathrm{n}=12\end{array}$ & origen & $\begin{array}{l}\text { Parte } \\
\text { usada }\end{array}$ & Uso \\
\hline $\begin{array}{l}\text { Maclura tinctoria (L.) D. } \\
\text { Don. ex Steud }\end{array}$ & mora & $1(0,06 \%)$ & 8,33 & $\mathrm{~N}^{*}$ & $\operatorname{Tr}$ & Lñ \\
\hline MORACEAE sp. 1 & sin nombre & $1(0,06 \%)$ & 8,33 & ¿? & & \\
\hline \multicolumn{7}{|l|}{ MUNTINGIACEAE } \\
\hline Muntingia calabura L. & nigüito & $4(0,22 \%)$ & 16,67 & $\mathrm{~N}$ & $\begin{array}{l}\mathrm{Cz}, \mathrm{Ex}, \mathrm{Fr} \\
\mathrm{Tr}\end{array}$ & $\begin{array}{l}\text { Ag-fe, Co-f, } \\
\text { C-m,Te-he, Te-pe }\end{array}$ \\
\hline \multicolumn{7}{|l|}{ MYRTACEAE } \\
\hline Psidium guajava L. & $\begin{array}{l}\text { guayaba, guayaba } \\
\text { dulce }\end{array}$ & $93(5,17 \%)$ & 91,67 & $\mathrm{~N}$ & $\begin{array}{l}\mathrm{Cg}, \mathrm{Cz}, \mathrm{Fr} \\
\mathrm{Hj}, \mathrm{Pl}, \mathrm{Tr}\end{array}$ & $\begin{array}{l}\text { Ag-fe, Co-f, Co- } \\
\text { b, Co-g, Co-d, } \\
\text { C-m,C-c, Lñ, Lu, } \\
\text { Te-co, Te-he }\end{array}$ \\
\hline Psidium guineense $\mathrm{Sw}$. & guayaba agria & $37(2,06 \%)$ & 75,00 & $\mathrm{~N}$ & $\mathrm{Fr}, \mathrm{Pl}, \mathrm{Tr}$ & $\begin{array}{l}\text { Co-f, Co-b, Co-d, } \\
\text { Lu, Or }\end{array}$ \\
\hline $\begin{array}{l}\text { Syzigium malaccense (L.) } \\
\text { Merr. \& L.M. Pery }\end{array}$ & pera & $18(1,00 \%)$ & 75,00 & I & Fr, Pl, Tr & $\begin{array}{l}\text { Co-f, Co-b, Co-d, } \\
\text { C-m,Me, Or, Te-he }\end{array}$ \\
\hline \multicolumn{7}{|l|}{ OXALIDACEAE } \\
\hline Averrhoa bilimbi $\mathrm{L}$. & curuba & $9(0,50 \%)$ & 33,33 & I & Fr & Co-b \\
\hline Averrhoa carambola $\mathrm{L}$. & corombolo & $5(0,28 \%)$ & 16,67 & I & Fr, Pl & Co-f, Co-b, Me, Or \\
\hline \multicolumn{7}{|l|}{ PHYLLANTHACEAE } \\
\hline $\begin{array}{l}\text { Phyllanthus acidus (L.) } \\
\text { Skeels }\end{array}$ & $\begin{array}{l}\text { droncella, } \\
\text { groncella }\end{array}$ & $3(0,17 \%)$ & 25,00 & I & Fr & Co-b, Co-d \\
\hline Phyllanthus elsiae Urb. & pimiento & $1(0,06 \%)$ & 8,33 & $\mathrm{~N}$ & $\mathrm{Pl}, \mathrm{Tr}$ & $\mathrm{Cv}, \mathrm{Lñ}$ \\
\hline \multicolumn{7}{|l|}{ POLYGONACEAE } \\
\hline Coccoloba acuminata Kunth & maiztostao & $1(0,06 \%)$ & 8,33 & $\mathrm{~N}^{*}$ & $\mathrm{Pl}$ & Or \\
\hline \multicolumn{7}{|l|}{ RUBIACEAE } \\
\hline Borojoa patinoi Cuatrec. & borojó & $1(0,06 \%)$ & 8,33 & I & $\mathrm{Fr}$ & Co-b \\
\hline Morinda citrifolia $\mathrm{L}$. & noní & $13(0,72 \%)$ & 41,67 & I & $\begin{array}{l}\mathrm{Fr}, \mathrm{Hj}, \mathrm{Pl}, \\
\mathrm{Tr}\end{array}$ & $\begin{array}{l}\text { Ag-fe, Ag-i, Cv, } \\
\text { Lñ, Me, Te-co, } \\
\text { Te-he }\end{array}$ \\
\hline Morinda royoc L. & Sin nombre & $1(0,06 \%)$ & 8,33 & $\mathrm{~N}$ & & \\
\hline \multicolumn{7}{|l|}{ RUTACEAE } \\
\hline Citrus auratium Risso & naranja & $11(0,61 \%)$ & 33,33 & I & $\begin{array}{l}\mathrm{Cz}, \mathrm{Fr}, \mathrm{Hj} \\
\mathrm{Tr}\end{array}$ & $\begin{array}{l}\text { Co-f, Co-b, Me, } \\
\text { Te-he }\end{array}$ \\
\hline Citrus medica $\mathrm{L}$. & limón & $4(0,22 \%)$ & 33,33 & I & Fr, $\mathrm{Hj}, \mathrm{Tr}$ & $\begin{array}{l}\text { Co-f, Co-b, Lñ, Lu, } \\
\text { Me, Te-co }\end{array}$ \\
\hline Citrus reticulata Blanco & mandarina & $22(1,22 \%)$ & 83,33 & I & $\mathrm{Fr}, \mathrm{Hj}$ & Co-f, Co-b, Me \\
\hline \multicolumn{7}{|l|}{ SAPINDACEAE } \\
\hline Melicoccus bijugatusJacq. & mamón & $27(1,50 \%)$ & 75,00 & $\mathrm{~N}$ & $\mathrm{Fr}, \mathrm{Pl}, \mathrm{Tr}$ & $\begin{array}{l}\text { Co-f, Co-b, Co-d, } \\
\text { C-m,Lñ, Or, As-f, } \\
\text { Te-he }\end{array}$ \\
\hline \multicolumn{7}{|l|}{ SAPOTACEAE } \\
\hline $\begin{array}{l}\text { Chrysophyllum argenteum } \\
\text { Jacq. }\end{array}$ & caimito & $2(0,11 \%)$ & 16,67 & $\mathrm{~N}$ & $\mathrm{Fr}$ & Co-f, Te-pe \\
\hline $\begin{array}{l}\text { Manilkara zapota (L.) Van } \\
\text { Royen }\end{array}$ & níspero & $10(0,56 \%)$ & 41,67 & $\mathrm{~N}$ & Fr, Se, Tr & $\begin{array}{l}\text { Co-f, Co-b, Co-d, } \\
\text { C-m,Me, Te-he }\end{array}$ \\
\hline
\end{tabular}


N. D. Jiménez-Escobar \& al., Huertos familiares en la bahía de Cispatá, Córdoba, Colombia

\begin{tabular}{|c|c|c|c|c|c|c|}
\hline Especies & Nombre común & Ind. & $\begin{array}{c}\text { Fr. } \\
n=12\end{array}$ & origen & $\begin{array}{l}\text { Parte } \\
\text { usada }\end{array}$ & Uso \\
\hline $\begin{array}{l}\text { Pouteria sapota (Jacq.) H.E. } \\
\text { Moore \& Stearn }\end{array}$ & Mamey & $1(0,06 \%)$ & 8,33 & $\mathrm{~N}$ & Fr, Tr & Co-f, Co-b, Lñ \\
\hline ZYGOPHYLLACEAE & & & & & & \\
\hline $\begin{array}{l}\text { Bulnesia arborea (Jacq.) } \\
\text { Engl. }\end{array}$ & guayacán de bola & $1(0,06 \%)$ & 8,33 & $\mathrm{~N}$ & $\operatorname{Tr}$ & $\mathrm{C}-\mathrm{m}, \mathrm{Lu}$ \\
\hline TOTAL & & $\begin{array}{c}1798 \\
(100.00 \%)\end{array}$ & 100 & & & \\
\hline
\end{tabular}

Numero de individuos y porcentajes (Ind.). Frecuencia relativa (Fr.) . Origen: Introducida (I), Nativa del departamento de Córdoba (N), Nativa en la Bahia de Cispata y presente en bosque estuarino o de manglar $\left(\mathrm{N}^{*}\right)$, De origen desconocido (¿?). Parte usada: Cogollos (Cg), Corteza (Cz), Exudados (Ex), Flores (Fl), Frutos (Fr), Hojas (Hj), Palmitos (Pl), Planta viva (Pv), Semillas (Se), Tronco/Tallos (Tr). Uso: Agropecuaria: abono (Ag-a), forraje (Agfo), insecticidas (Ag-i) y veterinario (Ag-v); Artesanal (Ar); Cercas vivas (Cv); Comestible: aceites (Co-a), bebidas (Co-b), condimentos (Co-c), dulces (Co-d), frutales (Co-f) y vegetales (Co-f); Construcción: ebanistería (C-e), cercas (C-c), maderable (C-m), no maderable (C-nm); Leña (Lñ); Lúdica (Lu); Mágico-religiosa (M-R); Medicinal(Me); Servicios Ambientales: alimento de animales silvestres (Sa-a); Ornamental (Or); Tecnológica: cosmético (Tc-co), herramientas (Tc-he) y pegantes (Tc-pe), pegantes para cazar aves (Tc-pc).

que se caracteriza por ser una especie común en los huertos de las regiones tropicales del mundo (Millat-E-Mustafa \& al., 1996; Wezel \& Bender, 2003; Albuquerque \& al., 2005; Florentino \& al., 2007; Pulido \& al., 2008; Beltrán, 2010), es muy apreciado en la región y se encuentra cultivado en la mayoría de huertos. Sin embargo, no se tuvo en cuenta en el presente trabajo por no ser considerado como un árbol entre los pobladores (por la ausencia de madera). La especie Terminalia catappa L. conocida como "almendro", es usada como ornamental en gran parte del Caribe colombiano, llegando a ser muy popular en los centros urbanos (Jiménez-Escobar \& Estupiñán-González, 2011), está presente en los huertos tropicales del mundo (RicoGray \& al., 1991; Millat-E-Mustafa \& al., 1996; Wenzel \& Bender, 2003), es un árbol reconocido por la habitantes de Cispatá y se puede observar creciendo en borde de caminos. No se le registró en ninguno de los huertos, porque actualmente algunas personas de la comunidad lo asocian a la mala suerte y a la desgracia, creencia que a tomado tal fuerza, que durante el trabajo de campo se pudo observar la tala de dos individuos por este motivo.

\section{Estructura de los huertos familiares}

Los huertos familiares en la bahía de Cispatá en su mayoría son de forma rectangular, delimitados por pequeños canales de agua y/o cercas vivas, varían entre $500 \mathrm{~m}^{2}$ a $5000 \mathrm{~m}^{2}$ (tamaño medio de $2496 \mathrm{~m}^{2}$ con una desviación estándar de $1429 \mathrm{~m}^{2}$; Tabla 2). Los huertos más grandes están delimitados por pequeñas zanjas que en época de lluvia se llenan de agua y establecen una vía fluvial en "chalupas" (pequeñas embarcaciones de madera), entre la comunidad y los manglares. En épocas de lluvias parte de los huertos se encharcan o se inundan.

Tabla 2. Tamaño de los huertos, numero de individuos y número de especies presentes en cada huerto familiar en la bahía de Cispatá, Córdoba, Colombia.

\begin{tabular}{lccc}
\hline \multicolumn{1}{c}{ Huerto } & Tamaño $\left.\mathbf{( m}^{\mathbf{2}}\right)$ & No. Ind & No. Sp \\
\hline H1 & 500 & 118 & 29 \\
H2 & 1000 & 50 & 16 \\
H3 & 1500 & 92 & 23 \\
H4 & 1700 & 63 & 25 \\
H5 & 1900 & 95 & 22 \\
H6 & 2100 & 49 & 26 \\
H7 & 2500 & 169 & 34 \\
H8 & 2500 & 130 & 33 \\
H9 & 2500 & 266 & 39 \\
H10 & 3750 & 250 & 34 \\
H11 & 5000 & 404 & 31 \\
H12 & 5000 & 112 & 25 \\
Promedio & 2496 & 150 & 28 \\
\hline TOTAL & $\mathbf{2 9 9 5 0}$ & $\mathbf{1 7 9 8}$ & $\mathbf{8 1}$ \\
\hline
\end{tabular}




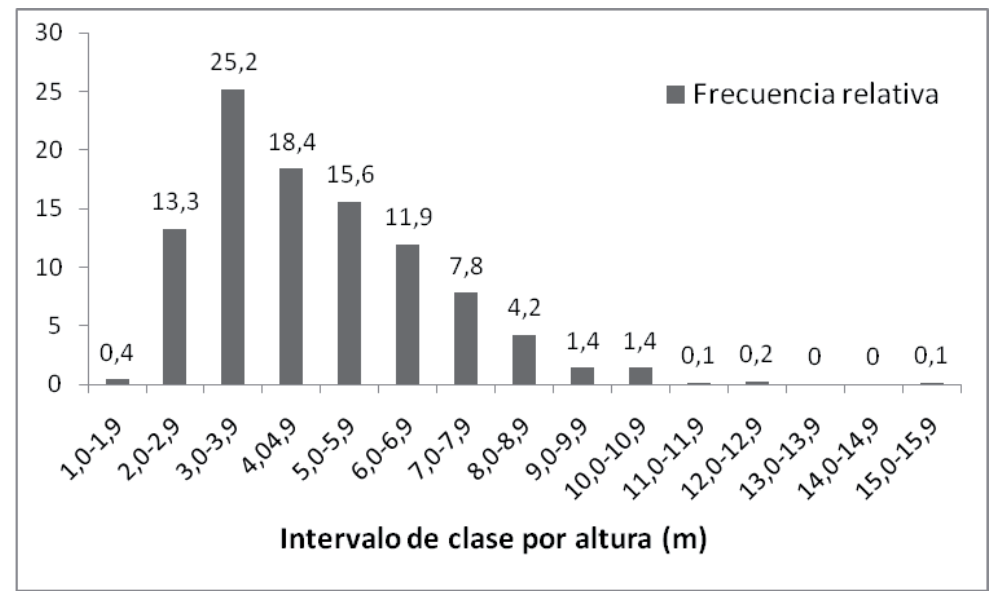

Fig. 2. Distribución de clases de los valores de altura para la vegetacion en huertos familiares en la bahía de Cispatá, Córdoba, Colombia.

Al igual que lo registrado por Pulido \& al. (2008) para otros huertos en Latinoamérica, la riqueza de especies no varía significativamente con el área de cultivo ( $\mathrm{r}: 0,5363 ; \mathrm{p}>0,05$ ). Sin embargo, se encontró una correlación significativa entre el área del huerto y el número de individuos ( $\mathrm{r}: 0,6439 ; \mathrm{p}<0,05$ ). Esto se explica porque los huertos más grandes no siempre presentan el mayor número de especies, pero si hay una tendencia a presentar mayor número de individuos.

En la vegetación de los huertos familiares se diferenciaron catorce clases de altura
(Fig. 2), el 92,2\% de los árboles y palmas se encuentran entre los 2 y los 7,9 m de altura y cerca del $60 \%$ se ubica en tan solo tres clases (tres, cuatro y cinco). Su distribución se aleja de la curva normal. Las especies de mayor altura son "pera" (Syzigium malaccense) y "roble" (T. rosea).

En el DAP se diferenciaron trece clases (Fig. 3), el 97,4 \% de las plantas medidas presentan diámetros inferiores a $40 \mathrm{~cm}^{2}$, siendo la primera categoría $(3,5-11,0 \mathrm{~cm})$ la que tiene mayor número de individuos. Las especies con los mayores diámetros fueron

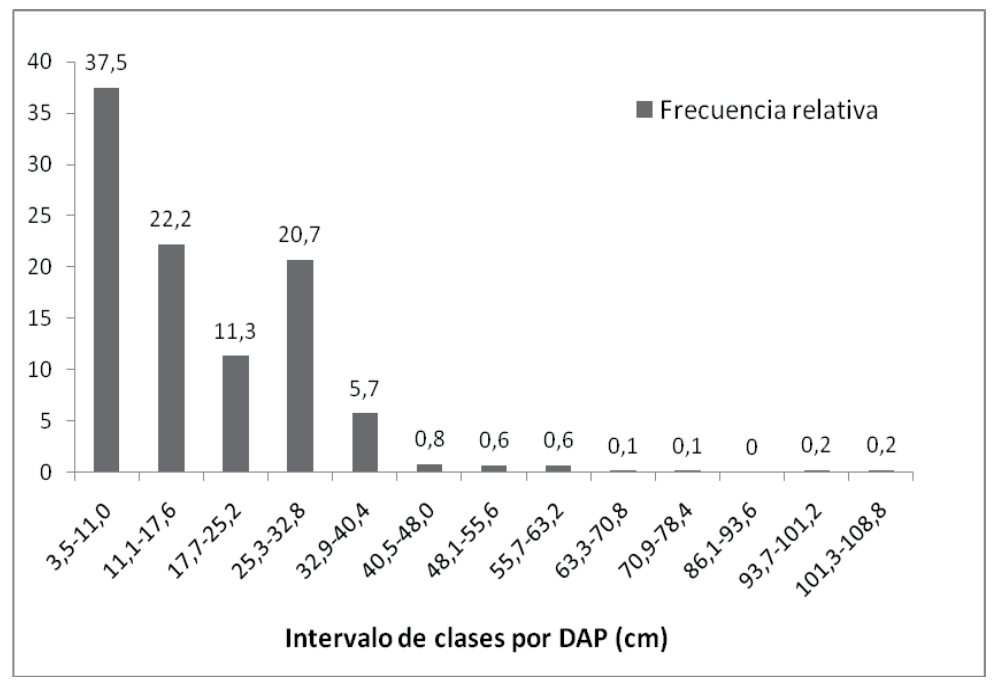

Fig. 3. Distribución de clases de los valores de DAP para la vegetacion de huertos familiares en la bahía de Cispatá, Córdoba, Colombia. 


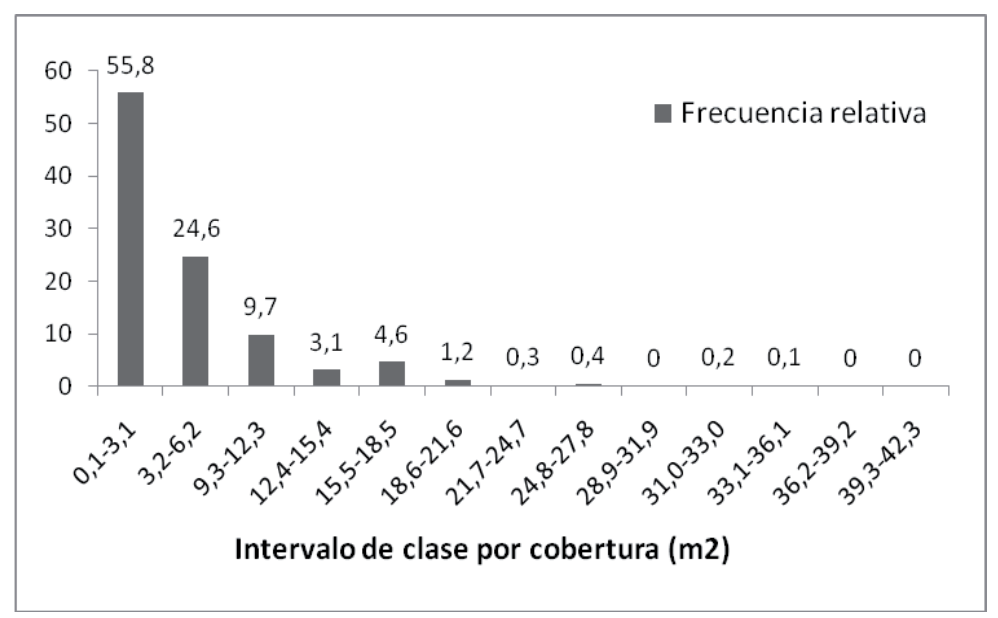

Fig. 4. Distribución de clases de los valores de cobertura para la vegetacion en huertos familiares en la bahía de Cispatá, Córdoba, Colombia.

E. oleifera $(108,8 \mathrm{~cm})$, seguida por $M$. indica $(106,5 \mathrm{~cm})$, estas dos especies son las únicas que se encuentran en las categorías de la ocho a la trece. La primera, corresponde a una palma cuyo mayor uso está enfocado a la producción de aceite de sus frutos y la extracción de sus hojas para hacer techos, ambas actividades no ponen en riesgo a los individuos. La segunda especie, es un frondoso árbol cuya madera puede ser usada como leña, en actividades de construcción y/o en la elaboración de tamboras, pero generalmente no es talado por la importancia de sus frutos en la región, lo cual les permite alcanzar amplios diámetros.

En la cobertura también se diferenciaron trece clases (Fig. 4), en la primera categoría se registraron más de la mitad de los individuos de las huertas familiares $\left(0,1-3,1 \mathrm{~m}^{2}\right)$. El "mango" ( $M$. indica) es la especie que presentó las mayores coberturas. Por lo general este árbol tiene un lugar privilegiado en el centro del huerto o en una de sus esquinas y no tiene que competir con otros árboles por luz. Otras especies que registraron las mayores coberturas son los maderables "orejero" (Enterolobium cyclocarpum), "roble" (T. rosea) y "caobo" (Swietenia macrophylla).

\section{Usos de los árboles en los huertos familiares}

De las 83 especies registradas en los huertos familiares, 26(31\%) están en una sola categoría de uso, $34(41 \%)$ entre dos y tres categorías de uso y $21(25 \%)$ cuatro o más categorías de uso. Sólo dos especies no presentan uso aparente (Moraceae sp.1 y Morinda royoc). La categoría que presentó mayor número de especies fue Comestible (46), seguida de Medicinal (34) y Construcción (33, Fig. 5).

Según la categoría de uso, la mayoría de los individuos podrían ser utilizados en Construcción (77,7\%), seguido de Tecnológica (73,9\%), Medicinal $(67,6 \%)$, Leña $(65,1 \%)$ y Comestible (53,2\%, Fig. 6). Por ser este un trabajo enfocado en árboles y palmas útiles, categorías como Construcción, Tecnológico y Leña ganan importancia. Sin embargo, los datos de diámetros y alturas no demuestran que estos individuos realmente estén siendo usados como productores de madera. Al contrario, son más utilizados en la construcción de varas para techos, cercas, elaboración de herramientas y en algunos casos como leña (principalmente en la época seca). Debido a esto, otras actividades parecieran quedar relegadas, alejándose de los patrones planteados por Pulido \& al. (2008) para otras huertas de Latinoamérica, donde el mayor número de individuos está relacionado con las categorías de uso Comestible, Medicinal y Ornamental.

Según Pulido \& al., (2008) las especies de los géneros Citrus, Musa, Inga, Psidium y Mangifera son frecuentes en los huertos de Latinoamérica, principalmente por su uso comestible. Estos géneros se encuentran 


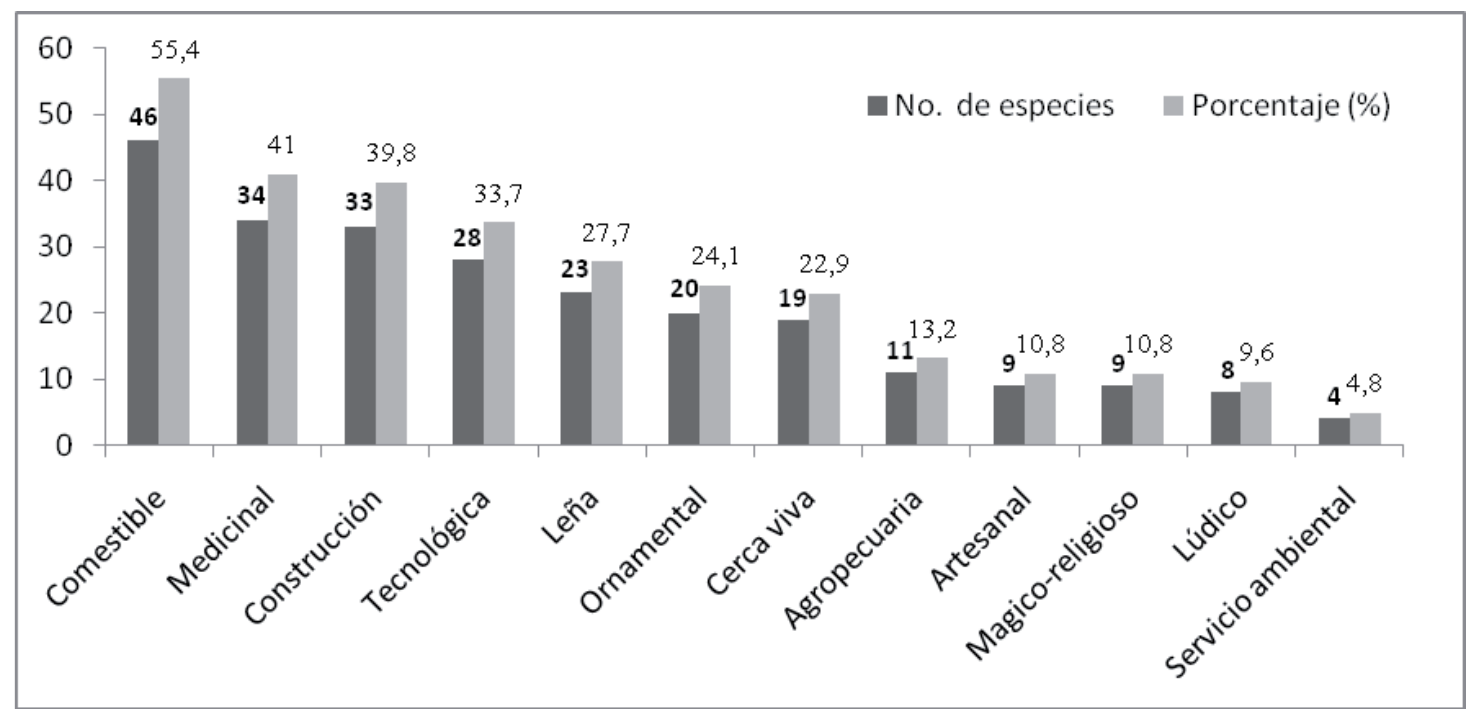

Fig. 5. Número de especies y porcentaje por categoría de uso de los árboles encontrados en huertos familiares en la bahía de Cispatá, Córdoba, Colombia.

representados en los huertos de la bahía de Cispatá, a pesar de que sus especies requieren altos suministros de agua y están enfrentadas a condiciones adversas como la salinidad y las prolongadas épocas de sequía o de inundación.

De las especies presentes en los huertos, la parte más usada corresponde al tronco
$(23,7 \%)$ que se relaciona con principalmente con las actividades dendroenergéticas y la elaboración de canoas, casas, cercas y herramientas, seguido por el fruto $(22,7 \%)$, que generalmente es usado para alimento. Toda la planta $(20,2 \%)$, que se refiere a tenerla viva dentro del huerto, se relaciona fundamentalmente con las actividades de

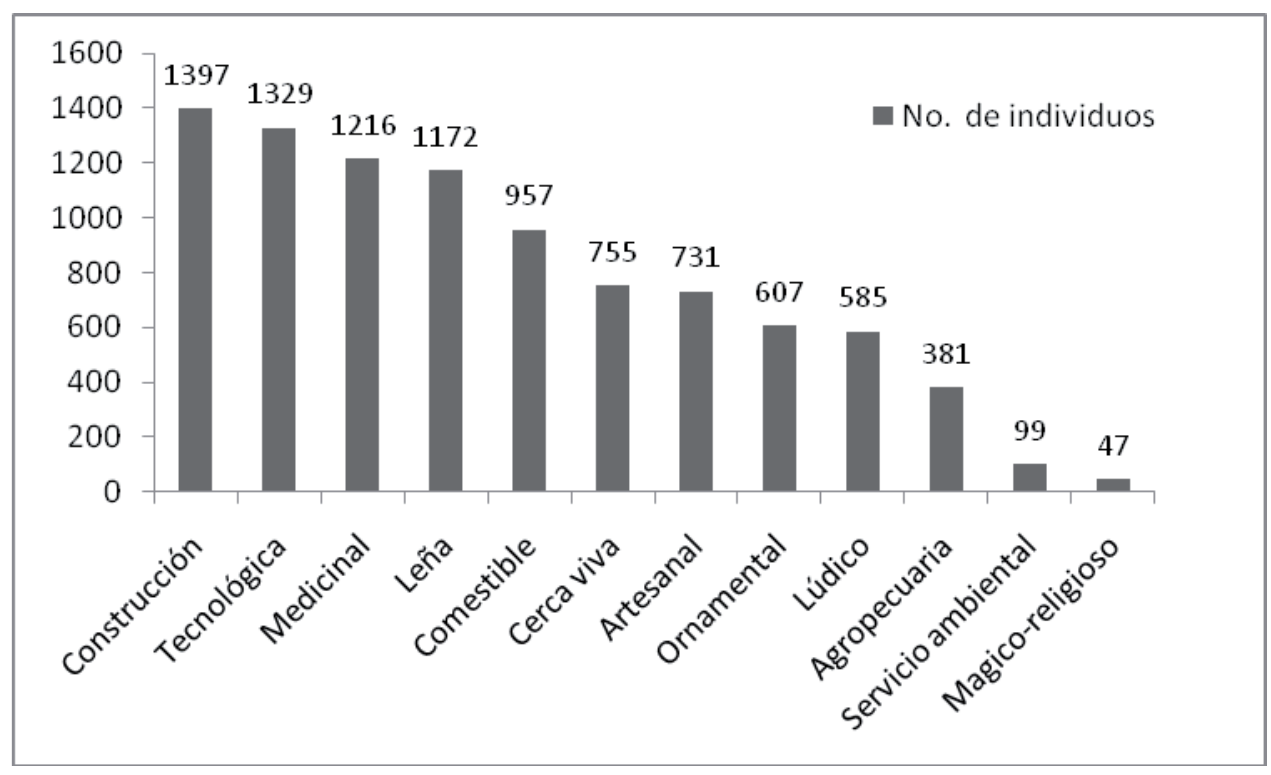

Fig. 6. Número de individuos por categoría de uso de los árboles encontrados en huertos familiares en la bahía de Cispatá, Córdoba, Colombia. 
cerca viva y ornamental y a su vez pueden prestar otros servicios como comestibles, leñas o maderables (Fig. 7).

Las especies que obtuvieron un mayor reconocimiento y que fueron nombradas dentro de la comunidad como los árboles o palmas más importantes por los 35 entrevistados fueron en su orden: $C$. nucifera, $M$. indica, $T$. rosea, C. odorata y P. guajava (Tabla 3 ).

Al separar los resultados de acuerdo con el género de los informantes, las mujeres citaron doce especies; las más importantes fueron C. nucifera, M. indica, P. guajava, T. rosea y Artocarpus cf. integrifolia. De estas cinco especies, cuatro son comestibles y las tres primeras que son las más citadas, hacen parte de la dieta básica y habitual de los pobladores. Por su parte los hombres nombraron dieciocho especies; las de mayor reconocimiento fueron T. rosea, C. odorata, $M$. indica, C. nucifera y $P$. guajava, de estas cinco, tres son comestibles, pero las dos especies más citadas por ellos corresponden a especies maderables. Toledo \& Barrera-Bassols (2010), consideran que al interior de las familias, el conocimiento se divide y se matiza de acuerdo con el género y la edad, debido a las actividades especificas que otorgan un conocimiento propio y particular. Estos resultados, reflejan el papel que juega el género en la distribución del conocimiento dentro de las familias en Caño Grande; las mujeres encargadas de la casa y del huerto, cuidan los hijos y los nietos, crían animales, cocinan y en general mantienen unido el núcleo familiar, razón por la cual dan prioridad a las especies comestibles, por encima de otras. Por su parte, los hombres en procura de bienestar económico para sostener a la familia, salen de la casa a cultivar y/o a pescar, dando especial atención a las especies maderables, con las que fabrican herramientas, viviendas, canoas y cercas.

La correlación entre el índice de Saliencia para las 21 especies nombradas y las abundancias de las mismas dentro de los huertos es significativa (r: 0,$6616 ; \mathrm{p}<0,05)$. $\mathrm{Si}$ sólo se tienen en cuenta las 17 especies presentes en los huertos, (r: 0,6958; $\mathrm{p}<0,05$ ) su correlación con el número de individuos también es significativa (Fig. 8).

Se diferenciaron dos especies nativas de los manglares y que no se cultivan en los huertos, pero su importancia cultural en la comunidad las destaca; estas fueron el "mangle colorado" (Rhizophora mangle) y el "mangle bobo" (Laguncularia racemosa). El "mangle colorado" es reconocido como la mejor leña de la región. Además, su madera es considerada

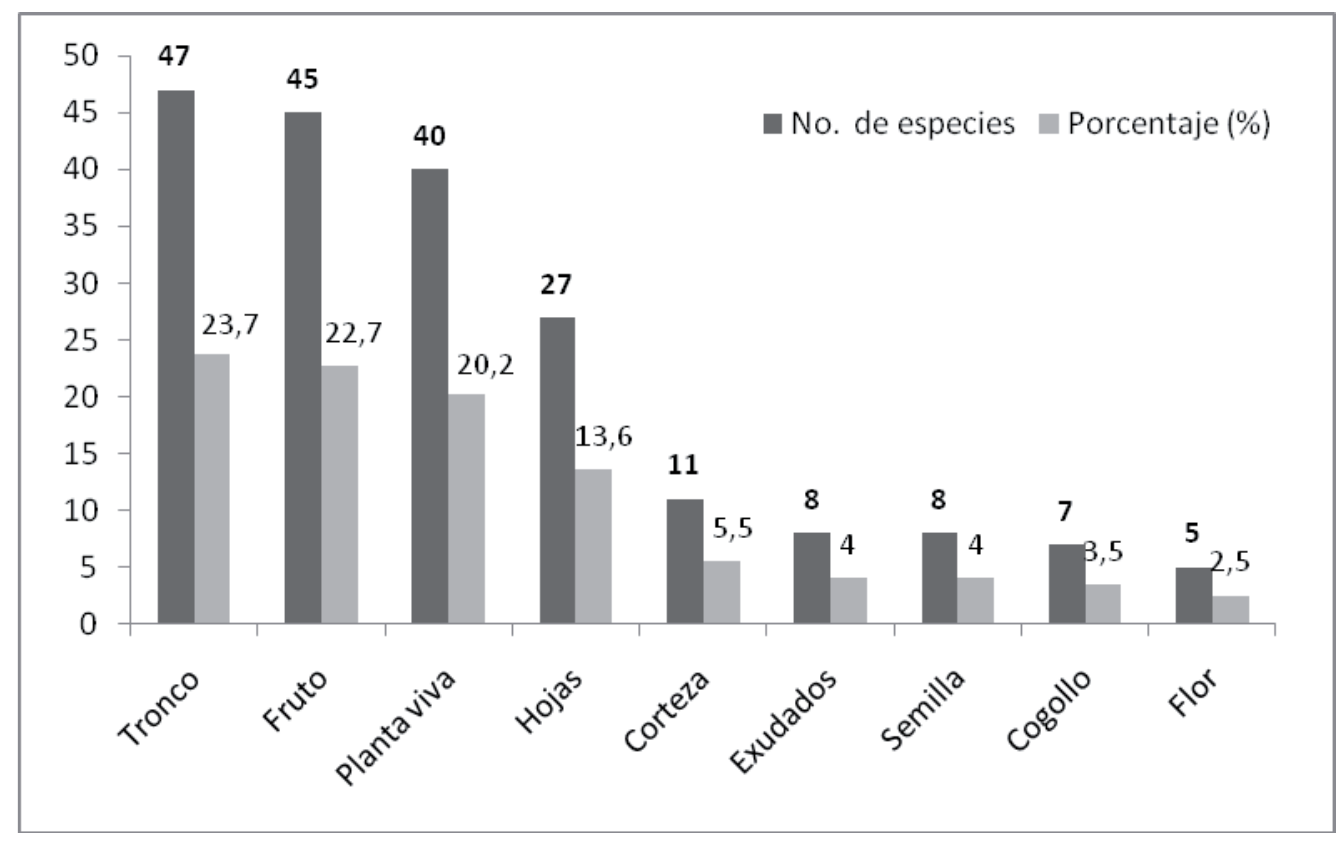

Fig. 7. Partes de la planta usada por una comunidad campesina en la bahía de Cispatá, Córdoba, Colombia. 
Tabla 3. Saliencia de las especies más importantes para la comunidad campesina de la bahía de Cispatá, Córdoba, Colombia.

\begin{tabular}{|c|c|c|c|}
\hline Especie & Nombre común & Frecuencia $(\%)$ & Saliencia \\
\hline Cocos nucifera & coco & 61,8 & 0,471 \\
\hline Mangifera indica & mango & 58,8 & 0,402 \\
\hline Tabebuia rosea & roble & 41,2 & 0,314 \\
\hline Cedrela odorata & cedro & 29,4 & 0,206 \\
\hline Psidium guajava & guayaba & 29,4 & 0,196 \\
\hline Sabal mauritiiformis & palma amarga & 14,7 & 0,118 \\
\hline Artocarpus cf. integrifolia & árbol pan & 11,8 & 0,039 \\
\hline Elaeis oleifera & palma de coroza & 5,9 & 0,039 \\
\hline Annona muricata & guanábana & 5,9 & 0,029 \\
\hline Persea americana & aguacate & 5,9 & 0,020 \\
\hline Rhizophora mangle * & mangle colorado & 5,9 & 0,020 \\
\hline Bactris guineensis & coroza & 2,9 & 0,029 \\
\hline Laguncularia racemosa* & mangle bobo & 2,9 & 0,020 \\
\hline Manilkara zapota & níspero & 2,9 & 0,020 \\
\hline Tectona grandis & teca & 2,9 & 0,020 \\
\hline Handroanthus chrysanthus* & guayacán & 2,9 & 0,010 \\
\hline Annona glabra & maguey & 2,9 & 0,010 \\
\hline Citrus auratium & naranja & 2,9 & 0,010 \\
\hline Eucaliptus sp.* & ocalipto & 2,9 & 0,010 \\
\hline Artocarpus altilis & pan de dios & 2,9 & 0,010 \\
\hline Carica papaya & papaya & 2,9 & 0,010 \\
\hline
\end{tabular}

* Especies no registradas dentro de los huertos.

de gran calidad para la construcción de viviendas y la elaboración de herramientas de uso cotidiano como: "cabos de hacha", "cucharas", "garabatos" (palo con forma de hoz que agrupa las espigas del arroz segundos antes de ser cortado), "molenillos" (palo cilíndrico en cuyo extremo se dejan algunos centímetros de las ramas más jóvenes a manera de aspa y que se usa para batir o revolver bebidas, preferiblemente el chocolate), "morteros" (instrumento de cocina usado para machacar achiote, ajo y algunas hierbas aromaticas), "palotes" (cuchara especial cuya única función es revolver el arroz), "palos de escoba", "pilones", "rastrillos" (herramienta en forma de $\mathrm{T}$ para extender e ir secando el arroz sobre un suelo de concreto antes de pasarlo por una trilladora que elimine el pericarpo), entre otros utensilios. El "mangle bobo" es un árbol de abundante follaje, muy usado como leña y en la fabricación de casas. Otras dos especies citadas entre las más importantes pero que no se encontraron en los huertos son: Handroanthus chrysanthus (Jacq.) S.O. Grose, un árbol de buena madera que crece en zonas abiertas y Eucaliptus sp., éste último es reconocido por sus usos medicinales, principalmente relacionados con el alivio de la gripa y otras enfermedades del sistema respiratorio, aunque según los pobladores el clima y la salinidad de los suelos no permiten que esta especie se establezca en sus territorios. La fama de su efectividad curativa, hace llegar sus hojas desde otros pueblos para ser vendidas en la bahía de Cispatá.

\section{Consideraciones finales}

En general los huertos de la bahía Cispatá están caracterizados por abarcar áreas entre $500-5000 \mathrm{~m}^{2}$, en promedio presentan 28 especies con cerca de 150 individuos. Los árboles dentro de los huertos son principalmente 


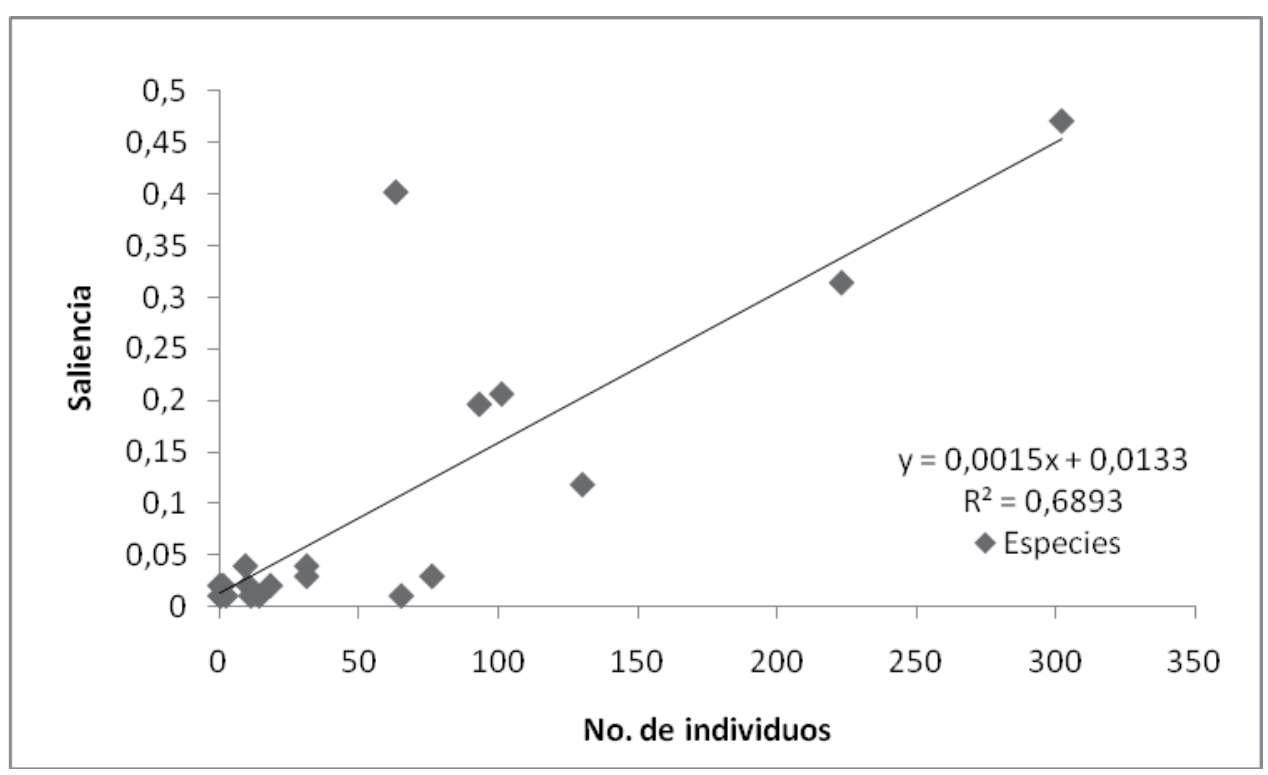

Fig. 8. Relación entre las especies más importantes y el número de individuos encontrados en los huertos familiares de la bahía de Cispatá, Córdoba, Colombia.

jóvenes con alturas entre 2-7 m, diámetros entre los 3,5-32,8 $\mathrm{cm}$ y coberturas entre 0,1 $18,5 \mathrm{~m}^{2}$. La palmera Cocos nucifera es la especie más importante para la comunidad, se encuentra en todos los huertos, presenta las mayores abundancias y culturalmente es la más reconocida por los pobladores.

Los huertos en esta región, suplen gran parte de las necesidades básicas de la comunidad, y en la actualidad son fundamentales para la seguridad alimentaria de los pobladores, acción que se refleja en la riqueza, la abundancia y la importancia cultural que le dan los pobladores a las especies comestibles.

Las huertas familiares además de proveer de productos fundamentales para el sostenimiento del núcleo familiar, son espacios vitales, generadores de procesos que fortalecen el tejido social. Es el lugar donde hay intercambio de productos agrícolas, juegan los niños y hay un flujo constante de saberes entre los pobladores (a nivel ambiental, cultural y social).

La relación positiva entre el número de individuos de una especie y su Saliencia cultural, puede sugerir que en los huertos familiares, las personas tienden a manejar y cultivar las especies que son más importantes culturalmente. El alto número de especies nativas demuestra que la composición de los huertos está ligada a factores culturales y de manejo, y que a su vez podría estar fuertemente relacionado con la disponibilidad del recurso en la zona.

A pesar de las condiciones de salinidad, los huertos familiares de la bahía de Cispatá presentan una riqueza de especies mayor a otros huertos del neotrópico, resaltándose su importancia como pequeños sistemas de diversidad que además albergan un alto porcentaje de especies nativas de la región. La documentación que se obtuvo sobre el uso de especies vegetales asociadas a las comunidades de manglar y de los bosques secos tropicales representa un paso importante hacia la conservación de estos ecosistemas. Especies nativas como: Annona glabra, Albizia saman, Bactris guineensis, Cedrela odorata, Elaeis oleifera, Handroanthus chrysanthus, Maclura tictorea, Sabal mauritiformis y Tabebuia rosea presentan un valor cultural importante en la comunidad y su inclusión en otros huertos de la región podría mitigar la deforestación de los bosques estuarinos y de manglar.

\section{Agradecimientos}

Al grupo de Biodiversidad y Conservación 
de la Universidad Nacional de Colombia, así como al Instituto de Ciencias Naturales y el Herbario Nacional Colombiano de la misma Universidad. Al Laboratorio de Etnobotánica Aplicada de la Universidad Federal Rural de Pernambuco (Brasil), especialmente al Ph.D. (c) Washington Soares. A la antropóloga María Celeste Medrano (Universidad de Buenos Aires-Argentina) por la compañía y la fortaleza durante las jornadas de campo. A la Dra. Lauren Raz por la revisión y las correcciones al manuscrito. A dos evaluadores anónimos por sus acertados comentarios. Nuestro más profundo agradecimiento a los pobladores de Caño Grande cuya alegría, apoyo, solidaridad y confianza permitieron realizar este trabajo. Esta contribución es derivada del proyecto "Valoración de la biodiversidad del Caribe colombiano: Síntesis del conocimiento y servicios ambientales (captura de $\mathrm{CO}^{2}$ )".

\section{Bibliografía}

ALBUQUERQUE, U.P., L.H.C. ANDRADE \& J. CABALLERO. 2005. Structure and floristics of homegardens in Northeastern Brazil. Journal of Arid Environments 62: 491-506.

— R.F.P. LUCENA\& N. L.ALENCAR. 2010. Métodos e técnicas para a coleta de dados etnobiologicos. En: U.P. Albuquerque, R.F.P. Lucena \& L.V.F. Cunha (orgs.). Métodos e técnicas na pesquisa etnobiologica e etnoecologica. Editora NUPEEA, Recife. 39-63 pp.

ÁlVAREZ-BUYLLA, M.E., E. LAZOS \& J.R. GARCÍA-BARRIOS. 1989. Homegardens of humid tropical region in Southeast Mexico: an example of an agroforestry cropping system in a recently established community. Agroforestry Sistems 8: 133-156.

AMOROZO, M.C.M, M. CULTRERA \& T. M. MIRANDA. 2008. Ethnobotanical studies in smallscale agriculture: local knowledge and maintenance of agricultural diversity. En: U. P. Albuquerque \& M.A. Ramos (eds.). Currents Topics in ethnobotany. Research Signpost. Recife. 55-79 pp.

AYRES, M., M.J. AYRES, D. L. AYRES \& S. A. SANTOS. 2007. Bioestat 5.0: Aplicações estatísticas nas áreas das ciências biológicas e médicas. Brasília, Sociedade Civil Mamirauá/CNPq.

BELTRÁN C., A.M. 2010. Etnobotánica de plantas medicinales de Guacamayas (Boyacá, Colombia). Trabajo de grado para optar al título de Bióloga, Instituto de Biología, Facultad de Ciencias Exactas y Naturales, Universidad de Antioquia. Medellín. $90 \mathrm{pp}$.

BORGATII, S. P. \& M. A. NATICK. 1996. Anthropac 4.0. Columbia: Analytic Technologies.

CORTÉS-CASTILLO. D.V. 2010. Flora y vegetación asociada a un gradiente de salinidad en el sector de bahía Cispatá (Córdoba-Colombia). Tesis de maestría. Departamento de Biología, Universidad Nacional de Colombia, Bogotá. 95 pp.

\& J. O. RANGEL-CH. 2011. Los bosques de mangle en un gradiente de salinidad en la bahía de Cispatá - Boca de Tinajones, Departamento de Córdoba - Colombia. Caldasia 33(1): 155-176.

- \& J. O. RANGEL-CH. En Imprenta. Flora y estructura de la vegetación estuarina en la bahía de Cispatá - Boca de Tinajones (Córdoba - Colombia). En: J.O. Rangel-Ch. (ed.). Colombia diversidad Biótica. Región Caribe (Vol XII).. Instituto de Ciencias Naturales, Universidad Nacional de Colombia-CORPOCESAR. Bogotá.

CRUZ, M.P., A.C. ESTUPIÑÁN-GONZÁLEZ, N.D. JIMÉNEZ-ESCOBAR, N. SÁNCHEZ, G. GALEANO \& E. LINARES. 2009. Etnobotánica de la región tropical del Cesar, Complejo Ciénaga de Zapatosa. En: J.O. Rangel-Ch. (ed.). Colombia diversidad biótica, media y baja montaña de la Serranía del Perijá (Vol VIII), Universidad Nacional de Colombia-Instituto de Ciencias NaturalesCORPOCESAR-REVIVE, Bogotá. 417-447 pp.

DANE. 2005. Departamento Administrativo Nacional de Estadística (DANE). [En línea] [Consultado 201011-11]. Disponible en: http://www.dane.org.co

ESTUPIÑÁN-GONZÁLEZ, A. C. \& N. D. JIMÉNEZESCOBAR. 2010. Uso de las plantas por grupos campesinos en la franja tropical del Parque Nacional Natural Paramillo (Córdoba, Colombia). Caldasia 32(1): 21-38.

, N. D. JIMÉNEZ-ESCOBAR, A. AVELLA-M. \& J. O. RANGEL-CH. 2011. Estructura y composición florística de los bosques inundables del Parque Nacional Natural Paramillo (Córdoba, Colombia) a. En: J. O. Rangel-Ch. (ed.). Colombia diversidad Biótica. Patrones de la estructura y de la riqueza de la vegetación en Colombia (VOL XI). Instituto de Ciencias Naturales, Universidad Nacional de Colombia. Bogotá D. C. 77 pp.

—, N. D. JIMÉNEZ-ESCOBAR, M. P. CRUZ, N. SÁNCHEZ, G. GALEANO \& E. LINARES. 2011. Plantas útiles del complejo cenagoso de Zapatosa. En: J. O. Rangel-Ch. (ed.). Colombia diversidad Biótica. Publicación especial No. 2. Guía de campo. Grupo de Biodiversidad y Conservación, Instituto de Ciencias Naturales, Universidad Nacional de Colombia-CORPOCESAR. Bogotá D. C. 77 pp.

FALS-BORDA, O. 1986. Retorno a la tierra: Historia doble de la Costa (Tomo IV). Carlos Valencia Editores. Bogotá. 234 pp. 
FAO. 2000. Food and Agriculture Organization (FAO). Mejorando la nutrición a través de huertos y granjas familiares: manual de capacitación para trabajadores de campo en América Latina y el Caribe. Dirección de Alimentación y Nutrición en colaboración con la Dirección de Producción y Protección Vegetal, Roma. [En línea] [Consultado 2011-05-11]. Disponible en: http://www.fao.org/ DOCREP/V5290S/V5290S00.htm

FLORENTINO, A.T.N., E.L. ARAÚJO \& U.P. ALBUQUERQUE. 2007. Contribuição de quintais agroflorestais na conservação de plantas da Caatinga, Município de Caruaru, PE, Brasil. Acta botanica brasilica 21(1): 37-47.

FRASER, J.A., A.B. JUNQUEIRA \& C. R. CLEMENT. 2011. Homegardens on Amazonian Dark Earths, Non-anthropogenic upland and floodsplain soils along the Brazilian middle Madeira river exhibit diverging agrobiodiversity. Economic Botany 65(1): $1-12$.

FRAUSIN, G., E. TRUJILLO, M. A. CORREA \& V. GONZÁLEZ. 2008. Seeds used in handicrafts manufactured by an Emberá-katío indigenous population displaced by violence in Colombia. Caldasia 30 (2): p.315-323.

GALEANO, G. 2000. Forest use at the Pacific Coast of Choco, Colombia; a quantitative approach. Economic Botany 54(3): 358-376.

HERNANDEZ, J. \& H. SANCHEZ. 1992. Biomas terrestres de Colombia. En: Halffter (Ed.) La diversidad biológica de Latinoamerica, CYTED-B, Programa Iberoamericano de Ciencia y Tecnología para el desarrollo, Instituto de Ecología, A.C. Secretaria de Desarrollo Social. México. 153-173 pp.

JIMÉNEZ-ESCOBAR, N.D., A.C. ESTUPIÑÁNGONZÁLEZ, N. SÁNCHEZ \& C. GARZÓN. 2009. Etnobotánica de la media montaña de la Serranía del Perijá. En: J. O. Rangel-Ch. (ed.) Colombia diversidad biótica, Media y baja montaña de la Serranía del Perijá (Vol VIII), Universidad Nacional de Colombia-Instituto de Ciencias NaturalesCORPOCESAR-REVIVE, Bogotá. 393-416 pp.

— \& A. C. ESTUPIÑÁN-GONZÁLEZ. 2011. Useful trees of the Caribbean region of Colombia. Bioremediation, Biodiversity and Bioavailability 5 (Special Issue 1): 65-79.

KARREMANS, J. 1994. Sociología para el desarrollo, métodos de investigación y técnicas de la entrevista. Serie técnica, informe técnico $\mathrm{N}^{\circ}$ 228. Centro Agronómico Tropical de Investigación y Enseñanza (CATIE). Turrialba, Costa Rica.

LAMONT, S.R., W.H. ESHBAUGH \& G. GREENBER. 1999. Species composition, diversity and use of homegardens among three amazonian villages. Economic Botany 53(3): 312-326.

LAWRENCE A., O.L. PHILLIPS, A. REATEGUI, M.
LOPEZ, S. ROSE \& D. WOOD. 2005. Local values for harvested forest plants in Madre de Dios, Perú: Towards a more contextualized interpretation of quantitative ethnobotanical data. Biodiversity and Conservation. 14: 45-79.

MARIN-CORBA, C., D. CARDENAS-LOPEZ \& S. SUAREZ-SUAREZ. 2005. Utilidad del valor de uso en etnobotánica. Estudio en el departamento de Putumayo (Colombia). Caldasia 27 (1): 89-101.

MILLAT-E-MUSTAFA, M.D., J. B. HALL \& Z. TEKLEHAIMANOTZ. 1996. Structure and floristics of Bangladesh homegardens. Agroforestry Systems 33: 263-280.

ORJUELA-R., M.A., J.O. RANGEL-CH. \& A. GARZON-C. 2004. Usos de las plantas en el Chocó biogeográfico. En: Rangel-Ch. (Ed.) Colombia diversidad biótica, El Choco biogeográfico / Costa Pacífica (Vol IV), Universidad Nacional de Colombia-Instituto de Ciencias Naturales, Bogotá. 911-936 pp.

ORTIZ F. 1989. Botánica medica Guahibo. Plantas medicinales, mágicas y psicotrópicas utilizadas por los Sicuani y Cuiba (Llanos orientales de Colombia). Caldasia 16: 14-22.

PADOCH, C. \& W. DE JONG. 1991. The house gardens of Santa Rosa: diversity and variability in an Amazonian agricultural system. Economic Botany 45(2): 166-175.

- 1996. Some quantitative methods for analyzing ethonobotanical knowledge. In Alexiades M.N. (Ed.). Selected guidelines for ethonobotanical research: a field manual. New York Botanical Garden. New York: 171-197

PULIDO, M.T., E.M. PEGAZA-CALDERON, A. MARTINEZ-BALLESTE, B. MALDONADOALMANZA, A. SAYNES \& R.M. PACHECO. 2008. Home gardens as an alternative for sustainability: Challenges and perspectives in Latin America. En: Albuquerque, U.P. \& M.A. Ramos (Eds.). Currents Topics in ethnobotany. Research Signpost. Recife. 55-79 pp.

RANGEL-CH, J.O. \& A. VELÁZQUEZ. 1997. Métodos de estudio de la vegetación. En: J.O. Rangel-Ch, P. Lowy \& M. Aguilar (eds.).Colombia. Diversidad biótica II: tipos de vegetación en Colombia. Instituto de Ciencias Naturales, IDEAM, Universidad Nacional de Colombia, Bogotá. 59-87 pp.

— \& H. ARELLANO-PEÑA. 2010. Clima. En: J. O. Rangel-Ch. (ed.). Colombia Diversidad Biótica IX, Universidad Nacional de Colombia (Instituto de Ciencias Naturales)-Corporación Autónoma Regional de los Valles del Sinú y San Jorge. Bogotá. $1-13 \mathrm{pp}$.

RENDÓN B., G. SOTO, M.I. OBLE, V. OJEDA, R.E. PARRA, E. PÉREZ \& L. RAMÍREZ. 1998. Theobroma bicolor Humb. \& Bonpl. (Sterculiaceae, "Cuapataxtle") en el municipio de Ayutla de los 
Libres, Guerrero. Composición florística de los huertos y aspectos etnobotánicos. Boletín de la Sociedad Botánica de México 63: 75-84.

RICO-GRAY, V., A. CHEMÁS \& S. MANDUJANO. 1991. Use of tropical deciduos forest species by Yucatecan Maya. Agroforestry Sistems 14(2): 149161.

RIVERA-DÍAZ, O. 2010. Flora. En: J. O. Rangel-Ch. (ed.). Colombia Diversidad Biótica, Ciénagas de Córdoba: Biodiversidad, Ecología y Manejo ambiental (Vol. IX), Universidad Nacional de Colombia-Instituto de Ciencias Naturales-Corporación Autónoma de los Valles del Sinú y San Jorge, Bogotá. 121-205 pp.

SÁNCHEZ, M. \& P. MIRAÑA. 1991. Utilización de la vegetación arbórea en el Medio Caquetá: 1. El árbol dentro de las unidades de la tierra, un recurso para la Comunidad Miraña. Colombia Amazónica 5(2): 69-98.

— P. MIRAÑA \& J. DUIVENVOORDEN. 2007. Plantas, suelos y paisajes: ordenamientos de la naturaleza por los indígenas Miraña de la Amazonía colombiana. Acta Amazónica 37(4): 567-582

SOKAL, R.R. \& F. J. ROHLF. 1995. Biometry. W.H. Freeman and Co. New York.

THE PLANT LIST. 2010. Version 1. [En línea]
[Consultado 2011-07-20]. Disponible en; http:// www.theplantlist.org/

TOLEDO, V. M. 1993. La Racionalidad Ecológica de la Producción Campesina. En: Ecología, Campesinado e Historia. Sevilla, E. y González de Molina, M. (editores). La Piqueta, Madrid: 197-218

_ \& N. BARRERA-BASSOLS. 2010. Aetnoecologia: uma ciência pós-normal que estuda as sabedorias tradicionais. En: V.A. Silva, A.L. Almeida \& U.P. Albuquerque (eds.). Etnobiologia e Etnoecologia, Pessoas \& Natureza na America Latina. NUPPEA. Recife. 13-36 pp.

TRUJILLO-C., W. \& M. CORREA-MUNERA. 2010. Plantas usadas por una comunidad indígena Coreguaje en la Amazonía colombiana. Caldasia 32 (1): $1-20$.

WEZEL, A. \& S. BENDER. 2003. Plant species diversity of homegardens of Cuba its significance for household food supply. Agroforestry Systems 57: 39-49.

W3TROPICOS. 2009. Missouri Botanical Garden VAST (VAScular Tropicos) nomenclatural database and associated authority files. [En línea] [Consultado 2011-01-08]. Disponible en: http://mobot.mobot. org/W3T/Search/vast.html

Original recibido el 1 de agosto de 2011; aceptado el 9 de diciembre de 2011. 